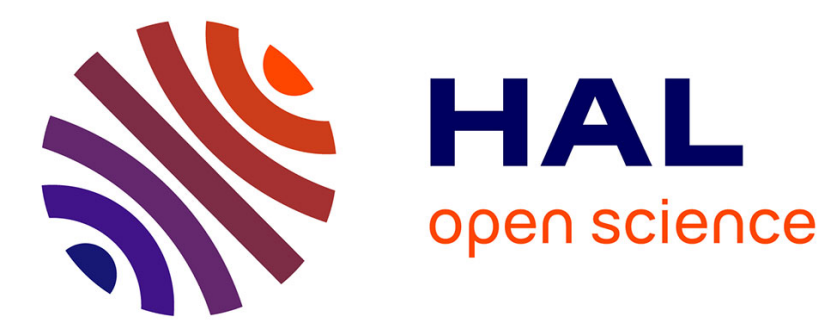

\title{
Fluid-structure interaction of a square cylinder at different angles of attack
}

Jisheng Zhao, Justin S. Leontini, David Lo Jacono, John Sheridan

\section{To cite this version:}

Jisheng Zhao, Justin S. Leontini, David Lo Jacono, John Sheridan. Fluid-structure interaction of a square cylinder at different angles of attack. Journal of Fluid Mechanics, 2014, Vol. 747, pp. 688-721. 10.1017/jfm.2014.167 . hal-01092016

\section{HAL Id: hal-01092016 https://hal.science/hal-01092016}

Submitted on 8 Dec 2014

HAL is a multi-disciplinary open access archive for the deposit and dissemination of scientific research documents, whether they are published or not. The documents may come from teaching and research institutions in France or abroad, or from public or private research centers.
L'archive ouverte pluridisciplinaire HAL, est destinée au dépôt et à la diffusion de documents scientifiques de niveau recherche, publiés ou non, émanant des établissements d'enseignement et de recherche français ou étrangers, des laboratoires publics ou privés. 


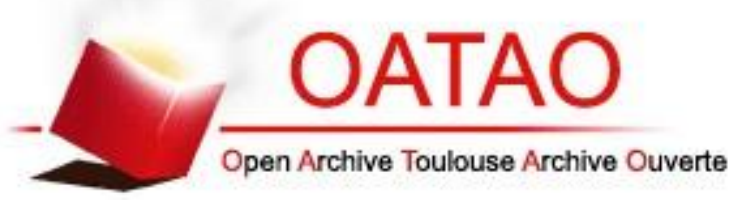

Open Archive Toulouse Archive Ouverte (OATAO)

OATAO is an open access repository that collects the work of Toulouse researchers and makes it freely available over the web where possible.

This is an author-deposited version published in: http://oatao.univ-toulouse.fr/ Eprints ID: 11815

Identification number: DOI : $10.1017 / \mathrm{jfm} .2014 .167$

Official URL: http://dx.doi.org/10.1017/jfm.2014.167

\section{To cite this version:}

Zhao, Jisheng and Leontini, Justin S. and Lo Jacono, David and Sheridan, John Fluid-structure interaction of a square cylinder at different angles of attack. (2014) Journal of Fluid Mechanics, Vol. 747 . pp. 688-721. ISSN 0022-1120

Any correspondence concerning this service should be sent to the repository administrator: staff-oatao@inp-toulouse.fr 


\title{
Fluid-structure interaction of a square cylinder at different angles of attack
}

\author{
Jisheng Zhao ${ }^{1,} \dagger$, Justin S. Leontini ${ }^{1,2}$, David Lo Jacono ${ }^{1,3}$ and \\ John Sheridan ${ }^{1}$ \\ ${ }^{1}$ Fluids Laboratory for Aeronautical and Industrial Research (FLAIR), Department of Mechanical and \\ Aerospace Engineering, Monash University, Melbourne, Vic 3800, Australia \\ ${ }^{2}$ Department of Mechanical Engineering and Product Design Engineering, Swinburne University of \\ Technology, John St Hawthorn, 3162, Australia \\ ${ }^{3}$ Institut de Mécanique des Fluides de Toulouse (IMFT), CNRS, UPS, Université de Toulouse, \\ Allée Camille Soula, F-31400 Toulouse, France
}

This study investigates the free transverse flow-induced vibration (FIV) of an elastically mounted low-mass-ratio square cylinder in a free stream, at three different incidence angles: $\alpha=0^{\circ}, 20^{\circ}$ and $45^{\circ}$. This geometric setup presents a body with an angle of attack, sharp corners and some afterbody, and therefore is a generic body that can be used to investigate a wide range of FIV phenomena. A recent study by Nemes et al. (J. Fluid Mech., vol. 710, 2012, pp. 102-130) provided a broad overview of the flow regimes present as a function of both the angle of attack $\alpha$ and reduced flow velocity $U^{*}$. Here, the focus is on the three aforementioned representative angles of attack: $\alpha=0^{\circ}$, where the FIV is dominated by transverse galloping; $\alpha=45^{\circ}$, where the FIV is dominated by vortex-induced vibration (VIV); and an intermediate value of $\alpha=20^{\circ}$, where the underlying FIV phenomenon has previously been difficult to determine. For the $\alpha=0^{\circ}$ case, the amplitude of oscillation increases linearly with the flow speed except for a series of regimes that occur when the vortex shedding frequency is in the vicinity of an odd-integer multiple of the galloping oscillation frequency, and the vortex shedding synchronizes to this multiple of the oscillation frequency. It is shown that only odd-integer multiple synchronizations should occur. These synchronizations explain the 'kinks' in the galloping amplitude response for light bodies first observed by Bearman et al. (J. Fluids Struct., vol. 1, 1987, pp. 19-34). For the $\alpha=45^{\circ}$ case, the VIV response consists of a number of subtle, but distinctly different regimes, with five regimes of high-amplitude oscillations, compared to two found in the classic VIV studies of a circular cylinder. For the intermediate $\alpha=20^{\circ}$ case, a typical VIV 'upper branch' occurs followed by a 'higher branch' of very large-amplitude response. The higher branch is caused by a subharmonic synchronization between the vortex shedding and the body oscillation frequency, where two cycles of vortex shedding occur over one cycle of oscillation. It appears that this subharmonic synchronization is a direct result of the asymmetric body. Overall, the FIV of the square cylinder is shown to be very rich, with a number of distinct regimes, controlled by both $\alpha$ and $U^{*}$. Importantly, $\alpha$ controls the underlying FIV phenomenon, as well as controlling the types of possible synchronization between the oscillation and vortex shedding.

Key words: flow-structure interactions, vortex streets

$\dagger$ Email address for correspondence: jisheng.zhao1@gmail.com 


\section{Introduction}

This study presents the results of experiments on the flow past an elastically mounted square-cross-section cylinder, where the flow is perpendicular to the long axis of the cylinder, and the cylinder is constrained to oscillate across the flow. As such, this setup falls under the broader class of investigations of the fluid-structure interaction of bluff, slender structures in cross-flow. This particular geometry is susceptible to two main flow-induced vibration (FIV) phenomena: vortex-induced vibration (VIV) where the frequency of periodic vortex shedding and the frequency of the body oscillation synchronize; and transverse galloping, an aeroelastic instability, caused by changes in the relative angle of attack induced by the body motion resulting in aerodynamic forces in the same direction as the motion. The prevalence and importance of FIV of such structures in practical engineering applications has motivated extensive investigations in the past half-century that aim to characterize, predict, and suppress FIV of bluff bodies. Comprehensive reviews of this large body of research work have been given by Blevins (1990), Naudascher \& Rockwell (2005) and Païdoussis, Price \& De Langre (2010).

The FIV of an elastically mounted body constrained to oscillate across the stream is dependent on the body mass in oscillation $m$, the mechanical damping $c$ and the spring stiffness $k$ (both assumed here to be constant), the fluid density $\rho$, the kinematic viscosity $v$, and the inflow speed $U$. A number of dimensionless groups can be defined, but typically for VIV studies the following independent parameters are used: the mass ratio, $m^{*}=m / m_{d}$, where $m_{d}$ is the mass of the fluid displaced by the body; the structural damping ratio with consideration of the added mass,

$$
\zeta=c /\left(2 \sqrt{k\left(m+m_{A}\right)}\right)
$$

in which $m_{A}$ is the added mass, which can be estimated from potential flow or measured directly through its influence on the natural frequency of the body in still fluid; the reduced velocity, $U^{*}=U / \sqrt{k\left(m+m_{A}\right)} H=U / f_{N} H$; and the Reynolds number, $R e=U H / \nu$. The displaced fluid mass is defined as $m_{d}=\rho C L$, where $C$ is the geometry's cross-sectional area, and $L$ is the length of the body immersed in the fluid. Here, $U$ is the free-stream velocity and $H$ is the length of the cross-section perpendicular to the flow. For this particular study, the angle of attack of the square cross-section with respect to the flow, $\alpha$, is also a variable.

A number of dependent variables are also presented non-dimensionally. The body displacement $y_{b}$ can be normalized by $H$. Forces, such as the lift force, are presented as non-dimensional coefficients, $C_{y}=F_{y} / 0.5 \rho U^{2} H L$. Throughout this paper, measured frequencies are presented normalized by the natural frequency in water, $f / f_{N}$. The frequency of the body oscillation $f_{y_{b}}^{*}$, the frequency of the total lift $f_{C_{y}}^{*}$, the frequency of the vortex lift $f_{C_{v o r t e x}}^{*}$ and the frequency of the drag $f_{C_{d}}^{*}$, are all non-dimensionalized in this manner. It should be noted that the vortex lift is defined as the component of the lift force that remains after subtracting the potential-flow added mass lift, following the method of Lighthill (1986).

A circular cross-section is not susceptible to galloping. Galloping relies on the aerodynamic forces being a function of the angle of attack of the body. Due to its symmetry, a cylinder does not have an angle of attack. A cylinder is therefore only susceptible to VIV, and has served as an ideal model for fundamental research on this phenomenon. Many of the previous results can be found in a series of review articles (Bearman 1984; Sarpkaya 2004; Williamson \& Govardhan 2004). 
The result of these studies most pertinent to the current study is that the flow response for light cylinders $\left(m^{*} \leqslant 10\right)$ can be broken down into four distinct regimes as a function of increasing $U^{*}$, typically referred to as branches (Khalak \& Williamson 1996; Govardhan \& Williamson 2000). First is the initial branch, where the amplitude of oscillation increases with $U^{*}$ and the oscillation is modulated due to the influence of both the body natural frequency and the Strouhal frequency (the vortex shedding frequency of a stationary cylinder). Second is the upper branch, characterized by oscillations of large amplitude at a frequency around the body natural frequency, that appear to be unstable and chaotic (Hover, Techet \& Triantafyllou 1998; Morse $\&$ Williamson 2009). The wake configuration in the upper branch consists of two oppositely signed pairs of vortices per oscillation cycle, where one vortex in the pair is much stronger than the other, and so is designated $2 P_{o}$, following the naming convention of Williamson \& Roshko (1988). Third is the lower branch, consisting of very periodic and stable oscillations at amplitudes around $0.6 D$, where $D$ is the cylinder diameter, and a frequency around the natural frequency of the body. The vortex shedding and body oscillation are synchronized at the same frequency. The wake again consists of two pairs of oppositely signed vortices, but this time more even in strength, and so it is designated $2 P$. Finally, the synchronization is lost and a desynchronized regime takes over, consisting of small oscillations at a fluctuating frequency around the Strouhal frequency.

Moving to the phenomenon of galloping, square-cross-sectional cylinders oriented with a flat face normal to the flow have been widely adopted as the canonical experiment. While Den Hartog (1932) first proposed a criterion for the onset of galloping of ice-covered cables, Parkinson \& Smith (1964) developed a very successful quasi-steady theory to predict the amplitude response of a square cylinder undergoing galloping. The theory is especially successful for relatively heavy and highly damped bodies, such as typically occurs in air, where the galloping oscillation frequency is much lower than the vortex shedding frequency.

For relatively light and lightly damped bodies, such as occur in water, the oscillation frequency and vortex shedding frequency are not necessarily as well separated, and the impact of the vortex shedding is more directly felt. Bearman et al. (1987) conducted experiments in a wind tunnel using a system with low damping, and found that, while the overall picture is similar to that described by the theory of Parkinson \& Smith (1964), a 'kink' region in the amplitude response occurred at $U^{*} \approx 6 \pi$, with a strong third-harmonic frequency of the body oscillation present in the transverse lift frequency components. Theoretical efforts to capture the effect of the vortex shedding on the galloping response have been made by Bokaian \& Geoola (1984), and a combined model for galloping and VIV developed by Corless \& Parkinson (1988, 1993), with limited success.

Much less attention has been paid to FIV of a square cylinder with variation of angle of attack. A definition sketch for this case is given in figure 1. A recent paper by Nemes et al. (2012) experimentally investigated the influence of angle of attack of a square cylinder with low mass-damping ratio on the body's FIV response in the same water channel facility as used for this study. An overall picture of the flow regimes that occur as a function of $\alpha$ and $U^{*}$ was presented. It was observed that a higher branch (HB) of amplitude response occurred over a range of angle of attack, $10^{\circ}<\alpha<22.5^{\circ}$, where the body oscillation amplitudes are considerably higher than those seen in the upper branch associated with VIV, but with an oscillation frequency locked onto approximately half of the Strouhal frequency. A numerical study from Zhao, Cheng \& Zhou (2013), which allowed motion in both the cross-stream and streamwise directions, found a similar high-amplitude response regime. 


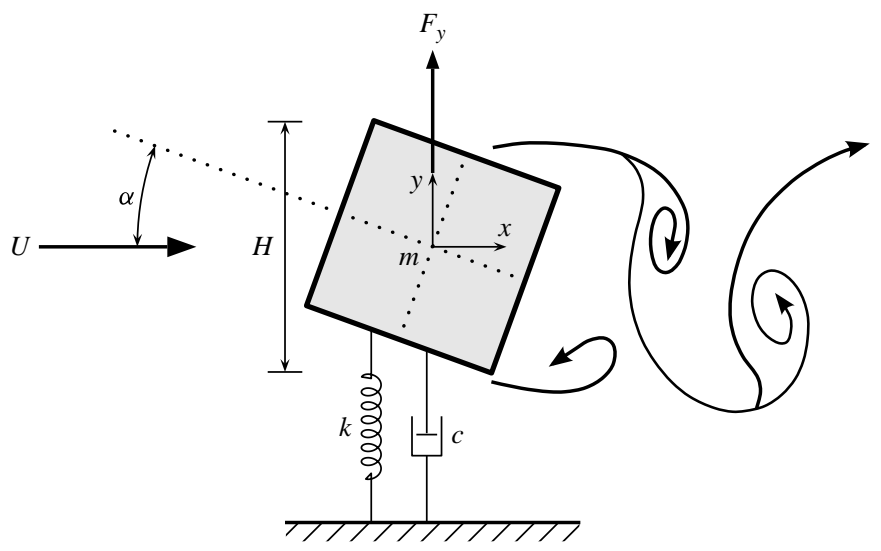

FIGURE 1. A definition sketch of the problem studied: a square cross-sectional cylinder with variable angle of attack $\alpha$, constrained to oscillate across the stream.

The present study aims to provide a precise and systematic description of the dynamics and response regimes of this system. As such, it significantly extends and refines the findings of Nemes et al. (2012). Careful experiments, at a fine resolution in terms of the independent parameters, allows different flow regimes to be clearly characterized and demarcated. As well as this, particle image velocimetry (PIV) and phase-averaging techniques have been employed for cases representing each of the identified flow regimes, to show the vorticity production and vortex wake configurations.

Three representative values of $\alpha$ have been chosen. The first, $\alpha=0^{\circ}$, is the canonical test case for transverse galloping, and so the findings can be compared directly with the results in the literature. The second, $\alpha=45^{\circ}$, results in FIV that is VIV dominated. As such, the results from this setup can be compared to the canonical results of VIV of a circular cylinder, and provide some insight into the generality of the circular cylinder results. The third, $\alpha=20^{\circ}$, presents the unique higher branch, and shows the impact of a geometry that breaks the reflection symmetry.

The experimental method, including the experimental apparatus, measurement techniques and experimental validation, is detailed in $\S 2$. The following section, $\S 3$, presents the obtained experimental results and discussion on the dynamic responses and wake modes of a freely vibrating square cylinder at $\alpha=0^{\circ}, 45^{\circ}$ and $20^{\circ}$. Lastly, conclusions of the present study are given in $\S 4$.

\section{Experimental method}

\subsection{Experimental apparatus}

The experiments were conducted in the free-surface recirculating water channel of the Fluids Laboratory for Aeronautical and Industrial Research (FLAIR), Monash University. More details of this water channel facility can be found in Sherry, Lo Jacono \& Sheridan (2010). The free-stream velocity in the present experiments was varied continuously in a range of $U=48-456 \mathrm{~mm} \mathrm{~s}^{-1}$.

The experimental setup is shown in figure 2. The rigid square cylinder model used in the present study was made from aluminium square-cross-sectional tubing with a side width of $24.6 \mathrm{~mm}$ and an immersed length of $L=620 \mathrm{~mm}$, giving an aspect ratio 
(a)

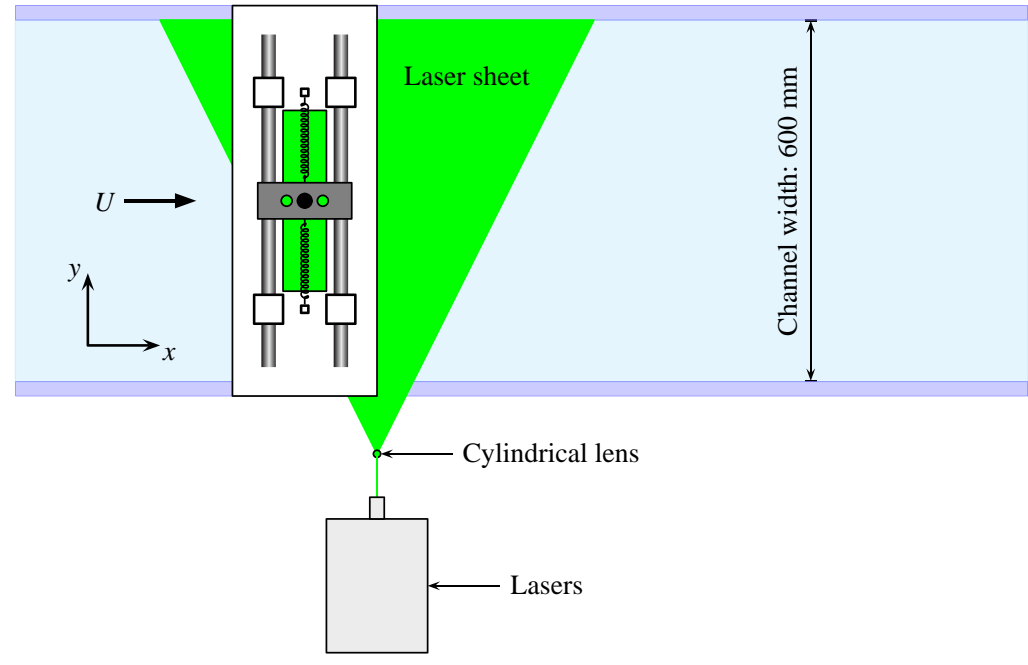

(b)
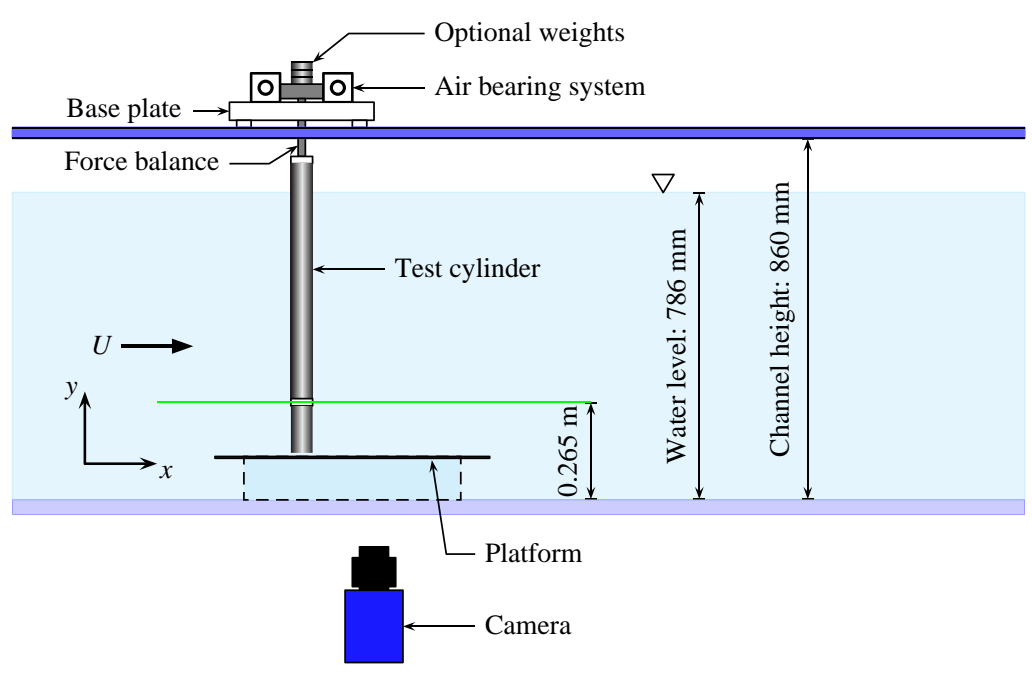

FIGURE 2. (Colour online) A schematic showing the experimental setup in the test section of the water channel: $(a)$ top view; $(b)$ side view.

range of $17.8 \leqslant A R=L / H \leqslant 25.2$. The displaced mass of water was $m_{d}=373.2 \mathrm{~g}$, giving a minimum achievable mass ratio of $m^{*}=2.64$ for the square cylinder model. In order to allow optical access the body was fitted with a $20 \mathrm{~mm}$ long Perspex section sitting $110 \mathrm{~mm}$ from the bottom end. The model was coupled with a force balance sting which was vertically mounted on an air bearing system. More details of this air bearing system can be found in Nemes et al. (2012). To promote parallel vortex shedding, an end conditioning platform technique used by Khalak \& Williamson (1997) was adopted in the present experiments. The present platform had a height of $165 \mathrm{~mm}$ and a top plate with dimensions of $600 \times 400 \times 5 \mathrm{~mm}$, giving a small gap of approximately $1 \mathrm{~mm}(4 \% H)$ between the cylinder free end and the water channel floor.

The natural frequencies of the system in both air and water were measured by conducting free decay tests individually in air and in quiescent water. The natural 
frequency in air was measured and assumed to be the natural frequency of the system in vacuum, while the natural frequency in water was also measured, for each angle of attack tested. The test results showed that the natural frequencies of the system for the three angles of attack were highly repeatable at $f_{N a}=0.803 \mathrm{~Hz}$ and $f_{N w}=0.648 \mathrm{~Hz}$. Thus, the structural damping ratio, defined as $\zeta=c /\left(2 \sqrt{k\left(m+m_{A}\right)}\right)=2.58 \times 10^{-3}$ was determined, in which $m_{A}=\left(\left(f_{N a} / f_{N w}\right)^{2}-1\right) m$.

\subsection{Measurements}

The displacement of the cylinder was measured using a non-contact magnetostrictive linear variable differential transformer (LVDT). The accuracy of the LVDT was within $\pm 0.01 \%$ of the $250 \mathrm{~mm}$ linear range available, giving a displacement measurement precision of $0.001 H$ (see Nemes et al. 2012). The lift and drag forces acting on the cylinder were measured simultaneously along with the cylinder displacement using a two-component force balance based on strain gauges configured in a Wheatstone bridge circuit. For the transverse lift measurement, the inertial force due to the cylinder's acceleration was taken into account to recover the instantaneous $F_{y}$ acting on the body using the following equation:

$$
F_{y}=F_{S G}+m_{B} \ddot{y}_{b}
$$

where $F_{S G}$ is the calibrated force output from the strain gauges of the lift measurement channel, $m_{B}$ is the bottom part of the system's mass consisting of half the force balance sting and the entire cylinder model, and $\ddot{y}_{b}$ is the body acceleration in the transverse direction.

Further insight can also be gained by decomposing this measured force into a component due to potential flow, and a component due to vorticity, which can be associated with the vortex shedding, according to the method proposed by Lighthill (1986), and employed by a number of studies of VIV (Govardhan \& Williamson 2000; Carberry, Sheridan \& Rockwell 2001, 2005). This gives two important time series of force: the total lift force $F_{y}$ and the vortex lift force $F_{\text {vortex }}$. The instantaneous relative phases between these two forces and the body displacement, $\phi_{\text {total }}$ and $\phi_{\text {vortex }}$, were calculated using the Hilbert transform (HT) (see Hahn 1996).

The vorticity fields in the near wake of the cylinder was measured using the PIV technique. The PIV system detailed in Nemes et al. (2012) was used for this purpose. The flow was seeded with hollow micro-spheres (model: Sphericel 110P8, Potters Industries Inc.) having a normal diameter of $13 \mu \mathrm{m}$ and a specific weight of $1.1 \mathrm{~g} \mathrm{~m}^{-3}$. Illumination was provided by two miniature $\mathrm{Ng}$ :YAG pulse lasers (Continuum Minilite II Q-Switched lasers) which produced a $2 \mathrm{~mm}$ thick horizontal planar sheet. Imaging was performed using a PCO 2000 (PCO, Germany) camera with a resolution of $2048 \times 2048$ pixel. The camera was equipped with a $50 \mathrm{~mm}$ lens (Nikon Corporation, Japan), giving a magnification of approximately 9.73 pixel $\mathrm{mm}^{-1}$ for the field of view of interest. In the present study, two phase-averaging methods were used. Where the body oscillations are highly periodic and sinusoidal (i.e. in the lock-in region of $\alpha=45^{\circ}$ ), the PIV measurements were triggered using a real-time control system at eight specified phases of the body's oscillations, giving 250 image pairs for each phase for phase-locked averaging. When the oscillations were periodic but not sinusoidal (i.e. in the cases of $\alpha=0$ and $20^{\circ}$ ), the PIV measurements were sampled at $4 \mathrm{~Hz}$ (more than eight times the body oscillation frequency for all $U^{*}$ ) initially at a random phase of the body motion. The cylinder's location at each PIV imaging trigger was determined by analysing 
the transistor-transistor logic (TTL) pulses and the LVDT signals, and then the PIV images were sorted into 12 different phase regions (or time intervals) with respect to the body oscillation period. To achieve high-quality phase-region-averaged PIV results, a large number of 5000 PIV image pairs were recorded for each reduced velocity of interest, yielding at least 400 image pairs for each of the 12 phase regions, which allowed much finer time intervals for phase-averaging compared to the method used previously in Nemes et al. (2012). The PIV image data were analysed with validated in-house PIV software developed by Fouras, Lo Jacono \& Hourigan (2008), using $32 \times 32$ pixel interrogation windows in a grid layout with $50 \%$ window overlap.

\subsection{Experimental validation}

The methodologies used are validated here against the published results of Khalak \& Williamson (1997) for VIV of a circular cylinder. The present circular cylinder had a diameter of $D=40 \mathrm{~mm}$ and an immersed length of $L=620 \mathrm{~mm}$, giving an aspect ratio of $A R=L / D=15.5$ and a displaced water mass of $m_{d}=780.1 \mathrm{~g}$. The total mass of the oscillating system was $m=1872.2 \mathrm{~g}$, resulting in a mass ratio of $m^{*}=2.40$. Free decay tests were conducted individually in air and water to determine the natural frequency in air, $f_{N a}=0.572 \mathrm{~Hz}$, and in water, $f_{N w}=0.477 \mathrm{~Hz}$. The structural damping ratio was measured at $\zeta=2.43 \times 10^{-3}$, resulting in a mass-damping ratio of $m^{*} \zeta=5.83 \times 10^{-3}$, which was comparable to the case study with $m^{*} \zeta=11.3 \times 10^{-3}$ (in which $m^{*}=2.4$ and $\zeta=4.5 \times 10^{-3}$ ) by Khalak \& Williamson (1997). The end condition was also controlled using the platform end control technique as described previously. The Strouhal number of the cylinder at rest of the present case is $S t=0.208$ which is in excellent agreement with $S t \approx 0.21$ for $1000<R e<10000$ consistently reported in the literature (see Norberg 2001).

The dynamic response of VIV was investigated over a reduced velocity of $2.75<$ $U^{*}<17$, corresponding to a flow velocity range of $49.5 \mathrm{~mm} \mathrm{~s}^{-1}<U_{\infty}<32.4 \mathrm{~mm} \mathrm{~s}^{-1}$ and a Reynolds number range of $2000<R e<13000$. The response amplitude and frequency of the present work are compared directly to the results of Khalak \& Williamson (1997) at the same mass ratio in figure 3. It should be noted that the results of Khalak \& Williamson (1997) include data of both increasing and decreasing reduced velocities for hysteresis and intermittency, while the present results include only data of increasing reduced velocities since the phenomena of hysteresis and intermittency are not focused on in this comparison. The comparison shows that the overall agreement is remarkable in terms of the three-branch amplitude response pattern, the amplitude peak value, the lock-in region, and the frequency response.

\subsubsection{The transverse lift force measurement}

As the transverse lift force is a crucial parameter in FIV studies, it is of importance to validate the lift measurement in experiments. However, very few such experimental validations can be found in the literature. For validation purposes, measurements of the lift acting on a circular cylinder undergoing VIV were also conducted.

The test cylinder used had a diameter of $25 \mathrm{~mm}$ and an immersed length of $620 \mathrm{~mm}$, resulting in a mass ratio of $m^{*}=2.64$. In particular, this diameter size was selected to validate that the force balance could measure the lift accurately for low force magnitudes. Figure 4 shows the results of the measured transverse lift forces $F_{y \text { (measured) }}$, determined by (2.1), compared with their calculated counterparts $F_{y(\text { calculated })}$, determined by the left-hand side of the equation of motion of the body,

$$
m \ddot{y}_{b}+c \ddot{y}_{b}+k y_{b}=F_{y(\text { calculated })}
$$



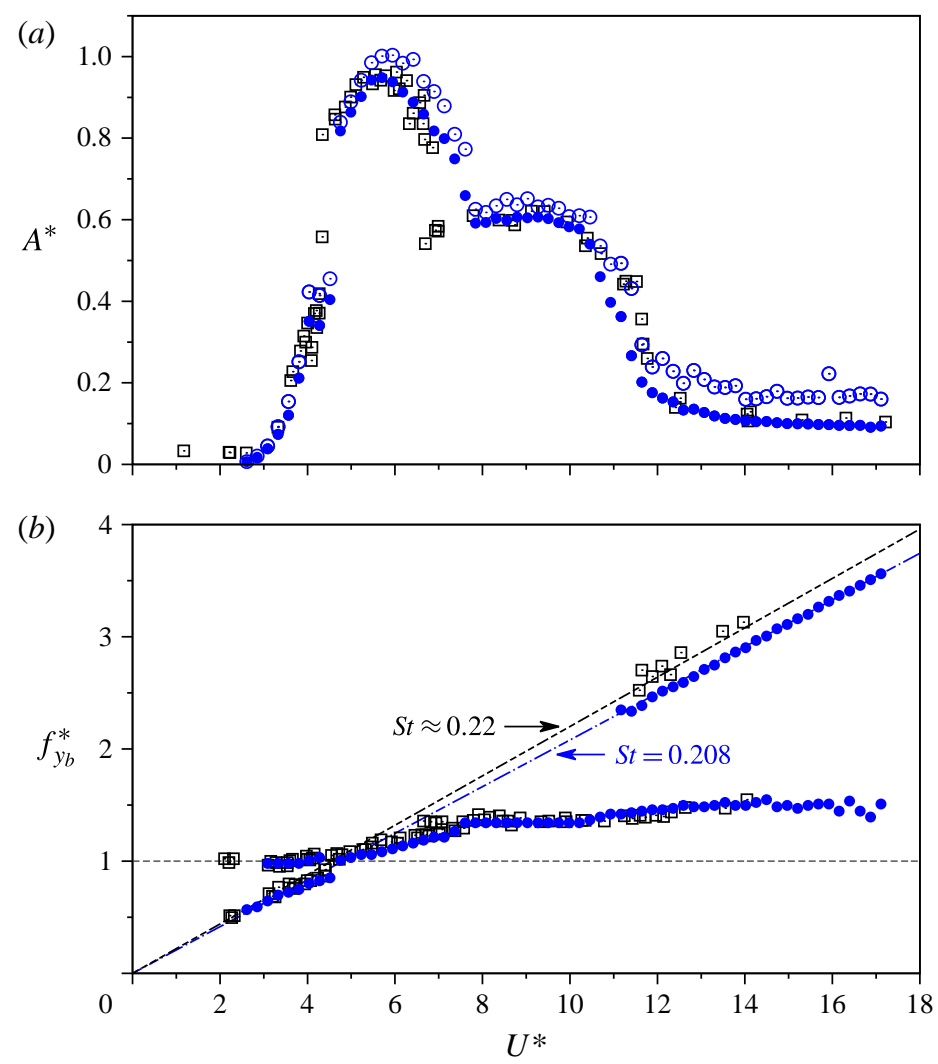

FIGURE 3. (Colour online) A comparison of experimental results shows excellent agreement between the present study and Khalak \& Williamson (1997) in $(a)$ the amplitude and $(b)$ the frequency responses of a circular cylinder with $m^{*}=2.4$. In $(a)$ open circles represent the normalized amplitude peaks, $A_{\max }^{*}$, of the present results, solid circles represent the present $A_{10}^{*}$ values, the mean of the top $10 \%$ of the oscillation amplitudes, and open squares represent the results of Khalak \& Williamson (1997). In (b) solid circles represent the present results, while open squares represent the results of Khalak \& Williamson (1997).

at different reduced velocities corresponding to the initial, the upper, and the lower branches. Note that in the figure, the two curves lie almost on top of each other, verifying that $F_{y \text { (measured) }}$ agreed well with $F_{y \text { (calculated) }}$ in a wide typical VIV range, indicating that the force balance could accurately measure the fluctuating $F_{y}$ at magnitudes as low as $0.005 \mathrm{~N}$.

\section{Results}

\subsection{Galloping-dominated response at $\alpha=0^{\circ}$}

The results presented in this section significantly expand upon previous results from Bearman et al. (1987) and Nemes et al. (2012). Using very fine increments of flow speed, represented by $U^{*}$, the previously reported kink is shown to be a region of harmonic resonance between the body oscillation and three cycles of the vortex shedding. Following the lead of Bearman et al. (1987), it is also shown that a similar 

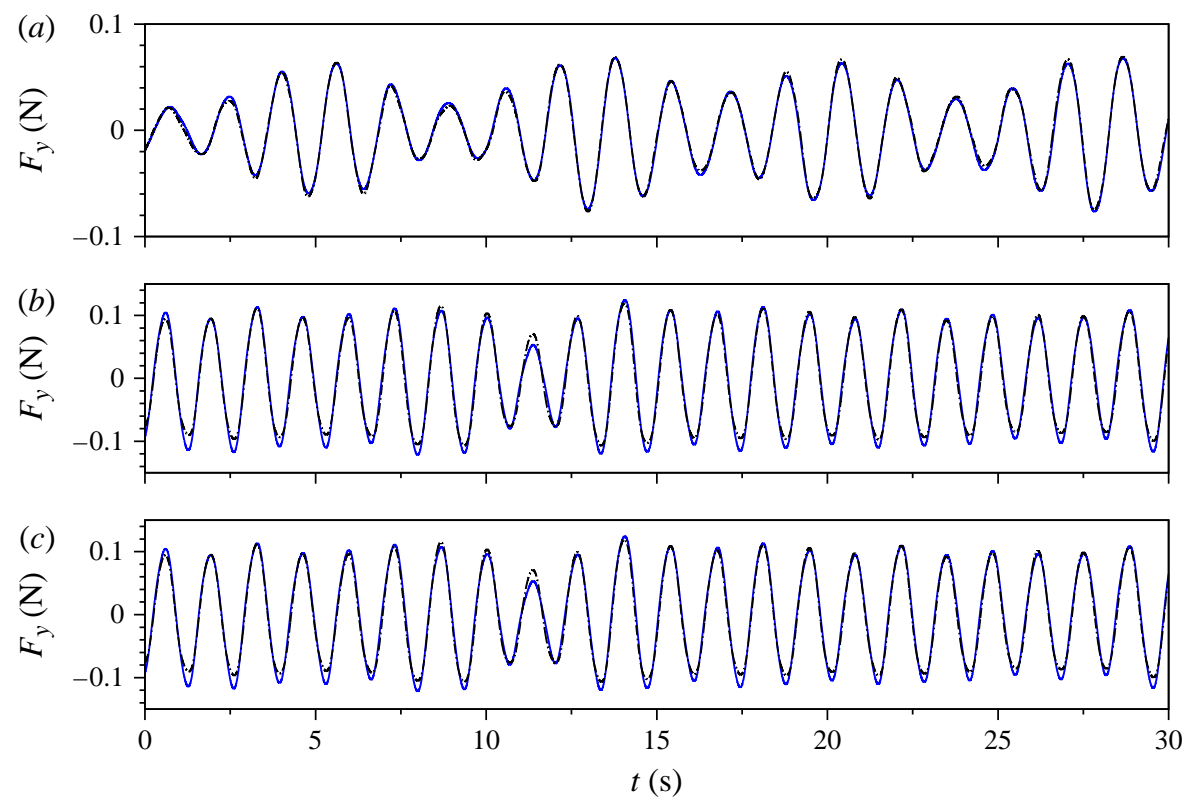

FIgURE 4. (Colour online) Time traces of the transverse lift force of a circular cylinder in the three typical response branches: (a) $U^{*}=4.4$, in the initial branch, $(b) U^{*}=5.0$, in the upper branch, and $(c) U^{*}=8.0$, in the lower branch. The solid lines represent the calculated values $\left(F_{y(\text { calculated })}\right)$ using the left-hand side of $(2.2)$, while the dashed lines represent the measurement values $\left(F_{y \text { (measured })}\right)$.

process occurs between the body oscillation and five cycles of vortex shedding. These synchronized regimes are clearly shown in the trend of the oscillation amplitude versus $U^{*}$, as well as in the dependence of the frequency content of the oscillation, lift force and drag force, on $U^{*}$. Establishing that the kink is due to resonance, as well as finding a series of synchronizations between the body oscillation frequency and odd numbers of vortex shedding cycles, appears to be a novel result.

Further, a novel explanation for the synchronization to odd numbers of vortex shedding cycles, while even numbers appear to have no special effect, is also provided.

\subsubsection{Amplitude and frequency response}

Figure 5(a) shows $A^{*}$ as a function of $U^{*}$ for the $\alpha=0^{\circ}$ case. Also shown (figure $5 b-e$ ) are contours of energy as a function of frequency and $U^{*}$ for four different sets of time series: the body oscillation, the total lift (transverse) force, the lift force attributable to the vortex shedding, and the total drag force. The contours were formed by taking the Fourier transform of the time series at a given value of $U^{*}$, then normalizing the resulting spectrum by the maximum energy, then 'stacking' the resulting normalized spectrum next to that obtained at the previous value of $U^{*}$. This normalization process means that the dominant frequencies at any value of $U^{*}$ are clearly visible on the plot, as are changes in the frequency response as a function of $U^{*}$. This process has been employed successfully previously by Assi, Bearman \& Meneghini (2010), Leontini, Lo Jacono \& Thompson (2011, 2013), Leontini \& Thompson (2013) and Tudball-Smith et al. (2012), the latter providing further details of how these plots are constructed. 

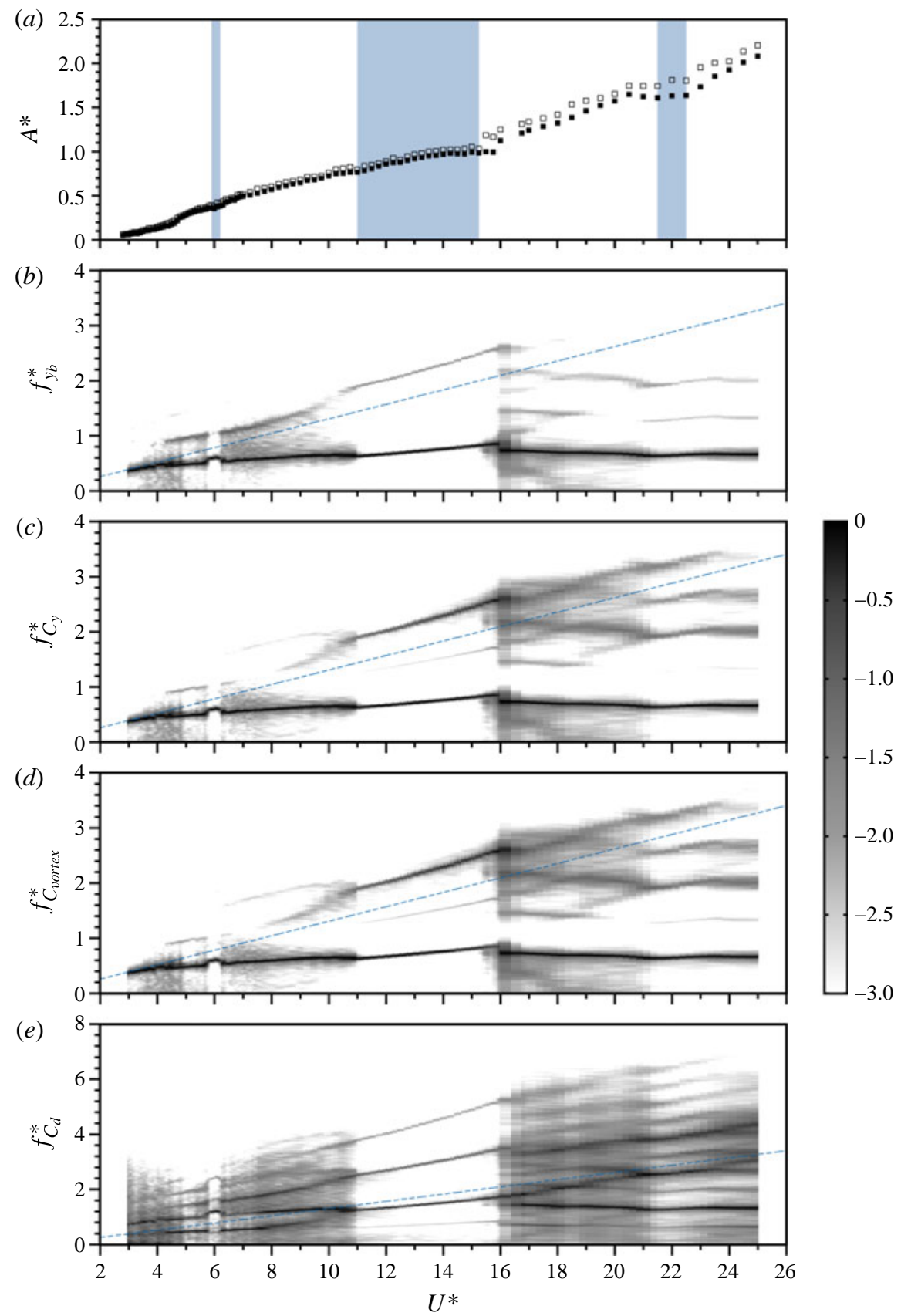

FIGURE 5. (Colour online) Three resonance regions are revealed in the amplitude response and frequency content as a function of reduced velocity of a free vibrating square cylinder with $m^{*}=2.64$ at $\alpha=0^{\circ}$. In $(a)$, open and solid squares represent $A_{\max }^{*}$ and $A_{10}^{*}$, respectively. The resonance regions are shaded in $(a)$, and correspondingly shown clearly by distinctive single-frequency response regions in $(b)-(e)$, which are the logarithmic-scale frequency power spectrum contour plots of the body oscillation $\left(f_{y_{b}}^{*}\right)$, transverse lift $\left(f_{C_{y}}^{*}\right)$, transverse vortex force $\left(f_{C_{\text {oortex }}}^{*}\right)$, and the drag $\left(f_{C_{d}}^{*}\right)$, respectively. Note that the frequency components are normalized by $f_{N w}$, e.g. $f_{y_{b}}^{*}=f_{y_{b}} / f_{N_{w}}$. The dashed line represents the value of St. 
While the general trend of classical galloping is shown in figure 5 ( $A^{*}$ increasing approximately linearly with $U^{*}$ once $U^{*}$ is beyond some threshold value), a number of features that diverge from the classical picture are immediately apparent. The most obvious are the steps or kinks in the amplitude response, that have been marked by the shaded background of figure $5(a)$.

The first of these kinks with increasing $U^{*}$ occurs around $U^{*}=6$. This coincides with a non-dimensional natural frequency $f_{N}^{*}=1 / U^{*} \simeq 0.167$. The Strouhal number for this system (the non-dimensional vortex shedding frequency from a rigidly mounted square) is $S t=0.131$. Therefore, this kink represents a small regime of VIV-dominated response, where the body motion frequency and vortex shedding frequency synchronize, and the motion is essentially periodic and regular. The motion in this regime is driven by the unsteady vortex shedding, as opposed to the long-time-average aerodynamic forces that dictate the motion during typical galloping. The synchronization between the oscillation frequency $f_{y_{b}}^{*}$ and the vortex shedding $f_{C_{\text {vortex }}}^{*}$ can be confirmed by comparing the spectra shown in figures $5(b)$ and $5(d)$. Both show the same single dominant frequency at $U^{*}=6$. The presence of only a single frequency also confirms the periodicity of the motion. As the frequency of the oscillation and the vortex shedding is the same, this is a 1:1 synchronization.

The second of these kinks, that occurs over a much larger range of $U^{*}$ than the first, is focused around $U^{*}=14$. This regime begins around $U^{*}=11$, and is marked by a small, but distinct plateau in the amplitude of oscillation. The regime ends around $U^{*}=16$, where there is a small but distinct increase in the amplitude of oscillation. Throughout this regime, the flow is periodic, with only a single dominant frequency occurring in the body oscillation $f_{y_{b}}^{*}$. This frequency increases very slowly, but essentially linearly, with $U^{*}$.

A key feature of this regime is the difference in the contribution of the frequency component at three times the body oscillation frequency, $3 f_{y_{b}}^{*}$, to the body oscillation and the lift force. For the body oscillation, the contribution of $3 f_{y_{b}}^{*}$ is relatively weak, whereas for the total lift force and the vortex lift force, both $f_{y_{b}}^{*}$ and $3 f_{y_{b}}^{*}$ make significant contributions. In fact, for the vortex lift force, the component at $3 f_{y_{b}}^{*}$ is the strongest component for $U^{*} \geqslant 14$. This is indicative of the fact that the vortex shedding is synchronized to $3 f_{y_{b}}^{*}$ in this regime, or a 1:3 synchronization. It appears that this regime is the same as the kink observed by Bearman et al. (1987).

The third of these kinks is focused around $U^{*}=22$. The regime is delineated by a region where the amplitude appears to be constant with increasing $U^{*}$. Similarly to the 1:1 synchronization and the 1:3 synchronization, this regime appears to have a single dominant frequency of oscillation $f_{y_{b}}^{*}$. However, the total and vortex lift spectra show a significant component at $5 f_{y_{b}}^{*}$, indicating that this regime is a 1:5 synchronization between the oscillation and the vortex shedding.

\subsubsection{Synchronization regimes}

As a whole, figure 5 shows that the FIV of a square cylinder generally follows the trends predicted by the quasi-static theory of transverse galloping developed by Parkinson \& Smith (1964), punctuated by a series of synchronization regimes between the body oscillation and the vortex shedding. Three key questions arise out of this picture: Why do the synchronization regimes occur at these particular values of $U^{*}$ ? Why do only 'odd' synchronizations occur, while 'even' ones do not (such as 1:2, 1:4, etc.)? What controls the extent of each of the synchronization regions?

The location of the first 1:1 synchronization can be understood by considering the interaction between the vortex shedding and the natural frequency of the body 
structure. When the vortex shedding frequency $f_{C_{\text {vortex }}}$ is close to the body natural frequency $f_{N}$, the body can respond favourably to the forcing provided. This response can then entrain the vortex shedding. This leads to oscillations where the body oscillation is synchronized with the vortex shedding to a frequency close to $f_{N}$, which is VIV. Therefore, this regime is expected near where $S t \simeq 1 / U^{*}$.

The location of the other synchronization regimes can also be understood by considering the behaviour of the body oscillation frequency and the vortex shedding frequency with increasing $U^{*}$. Figure $5(b)$ shows that over the entire range of $U^{*}$ tested, the primary body oscillation frequency $f_{y_{b}}$ remains essentially constant. Only in the synchronization regimes does it vary noticeably, and even then only by a small amount. However, when the Strouhal number remains constant the vortex shedding frequency $f_{C_{\text {vortex }}}$ is a linearly increasing function of the flow speed. Therefore, it would be expected that the vortex shedding frequency $f_{C_{\text {vortex }}}$ will be an integer multiple of the body oscillation $f_{y_{b}}$ at equi-spaced increments of $U^{*}$. Figure 5 shows the synchronization regimes occurring at around $U^{*}=6, U^{*}=14$, and $U^{*}=22$, which are indeed equi-spaced increments. Around these values, nonlinear synchronization causes the body oscillation and the vortex shedding to move from their natural values and synchronize at the values reported in figure 5.

Regardless of this synchronization, the relevant FIV phenomenon is still transverse galloping. This fact can be used to explain why only odd synchronizations occur, if it is assumed that the vortex shedding mode remains essentially the same as for the Kármán vortex street, i.e. one vortex shed from one side of the body during one halfcycle, followed by a vortex of opposite sign shed from the opposite side of the body in the next half-cycle.

Transverse galloping relies on the body being driven by the average transverse force over some time interval that is longer than the vortex shedding period. During the upstroke, the mean lift force must also be up (positive); during the downstroke, the mean lift must be down (negative). If a 1:2 resonance were to occur, one cycle of vortex shedding would occur during one half-cycle of the body oscillation. One cycle of vortex shedding from a nominally symmetric body gives a mean lift of zero, hence the vortex shedding cannot have an appreciable impact on the aerodynamic forces, and hence it cannot lead to a significant resonance (note that this is not necessarily the case when the body is not symmetric, as the strength of each vortex on each side of the wake can be different). On the other hand, during a 1:3 resonance, one and a half vortex shedding cycles occur during one half-cycle of oscillation, which certainly will have a mean lift, and hence this can have a significant impact on the forces and hence the motion.

For small ratios such as 1:3, it is also likely that the mean lift force will be different to that measured on a static body at an angle of attack, which is used in the quasistatic theory of galloping. Hence it might be expected that even though the oscillation is still driven by galloping, the response will differ from that of typical galloping, and this is indeed what is observed in figure 5.

The discussion above highlights that each synchronization region consists of the vortex shedding being locked to a multiple of the body oscillation frequency. This means that as the multiple gets higher, the number of vortices involved in the synchronization increases. Intuitively, it seems that as the number of vortices involved increases, the tuning required to obtain a locked, synchronized state becomes finer, and the range of $U^{*}$ over which the synchronization exists decreases. Indeed for simple maps, Cvitanović, Shraiman \& Söderberg (1985) showed theoretically that for synchronizations of the form 1:N, the stability interval (here the range of $U^{*}$ over 

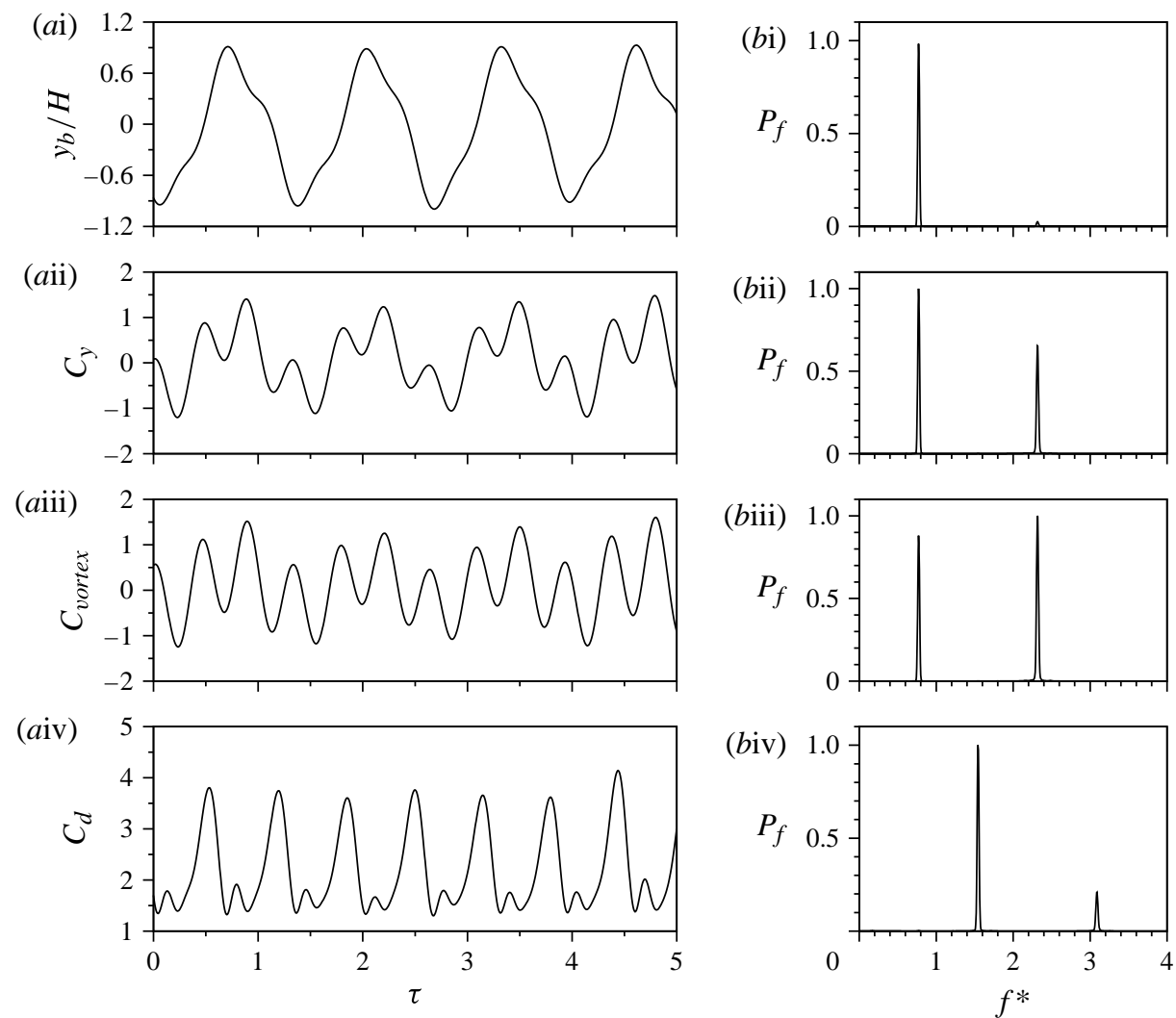

FIGURE 6. An example of the dynamics of the $1: 3$ synchronization for $\alpha=0^{\circ}$ : time traces (a) and normalized power spectra (b) of displacement (i), total lift (ii), vortex lift (iii) and drag (iv). The reduced velocity of this case is $U^{*}=14.26$. A third-harmonic frequency of the body oscillation is clearly seen in $C_{y}$ and $C_{\text {vortex }}$. The frequency components are normalized by $f_{N w}$, i.e. $f^{*}=f / f_{N w}$, and $\tau=t f_{N w}$.

which the synchronization exists) scales with $N^{-3}$. Excluding the 1:1 synchronization (which is controlled by VIV, not galloping), a significant reduction in the stability interval is observed with increasing $N$, with the 1:3 synchronization occurring over a much greater range of $U^{*}$ than the 1:5 synchronization.

It should also be noted that the same basic phenomena control the flow whether the flow is synchronized or not. These are the body oscillation, and the vortex shedding. Outside of the synchronization regimes, these two phenomena are uncorrelated, and so both contribute to the spectral content of the response, resulting in a modulated body oscillation. However, the two phenomena cannot be thought of as purely additive due to the nonlinearity of the system, and it is this nonlinearity that allows the body oscillation and vortex shedding to synchronize.

\subsubsection{Further details of the 1:3 synchronization}

This section presents details of the case at $U^{*}=14.26$, close to the centre of the range over which the 1:3 synchronization occurs as shown in figure 5 .

Figure 6 shows time traces and power spectra of the displacement and the fluid forces for the case at $U^{*}=14.26$. At this reduced velocity, the body's oscillations are 
highly periodic as shown in figure 6(ai), with a dominant frequency of $f_{y_{b}}^{*}=0.77$ and a third-harmonic frequency $\left(3 f_{y_{b}}^{*}=2.31\right)$ with a much weaker power magnitude as shown in figure $6(\mathrm{bi})$. The fluid forces $\left(C_{y}, C_{\text {vortex }}\right.$, and $\left.C_{d}\right)$ are also highly periodic. The drag is dominated by a frequency equal to the second-harmonic frequency of the body motion, and also has a component at twice this frequency. The total lift force fluctuates with the same frequency components as the body motion, however its third-harmonic frequency is much stronger in power than that of the body motion. This is reflected in the significant modulation of the time trace of the lift. If only the vortex lift is considered, the force is dominated by its third-harmonic frequency (while still possessing a significant component at the body-motion dominant galloping frequency). This indicates that the vortex shedding frequency is three times the galloping oscillation frequency.

For the 1:3 synchronization to be described as a modified galloping phenomenon, the lift force and the body oscillation should be essentially in phase. However, because of the reasonably complicated relationship between the lift force and the body oscillation, defining a phase between the two signals can be difficult. What is most important is the phase between the component of the lift force that is synchronized to the body motion. Rather than decompose the signal using a Fourier decomposition which assumes sinusoidal modes, the total lift and the vortex lift can be decomposed using empirical mode decomposition (EMD) pioneered by Huang et al. (1998). This results in mode shapes that are not sinusoidal, but they are orthogonal.

Figure 7 shows that the leading EMD mode of both $C_{y}$ and $C_{\text {vortex }}$, denoted by $C_{y(E M D 1)}$ and $C_{\text {vortex }(E M D 1)}$ respectively, oscillate with a frequency similar to the dominant frequency of the body motion. Therefore, the leading EMD modes of $C_{y}$ and $C_{\text {vortex }}$ can be thought of as representing the body oscillation. The second EMD modes of both $C_{y}$ and $C_{v o r t e x}$, denoted by $C_{y(E M D 2)}$ and $C_{\text {vortex (EMD2) }}$ respectively, oscillate with a frequency similar to the vortex shedding frequency, and can be thought of as representing the influence of the vortex shedding. For a galloping-dominated process, the long-time forces (over the period of body oscillation) are expected to be in phase with the oscillation. Figure $7(c)$ shows that this is indeed the case, further suggesting that the 1:3 synchronization is a galloping-dominated phenomenon.

Interestingly, figure $7(d)$ shows that the second EMD modes of the oscillation and the vortex shedding are also in phase. $C_{\text {vortex }(E M D 1)}$ is in phase with $C_{y(E M D 1)}$, shown in figure $7(c)$, and $d$ ). Thus, this EMD analysis of the lift and vortex forces further suggests that the 1:3 synchronization is a galloping-dominated phenomenon.

Images of the wake show further evidence of this. Figure 8 shows a series of twelve images of vorticity contours over one cycle of oscillation obtained from PIV. The images were obtained by averaging a series of images obtained over a small time period, as explained in $\S 2.2$. The time period averaged over for each image is marked on the time history plot in figure $8(a)$.

The images show very clearly the regular vortex shedding that occurs. The wake mode is essentially the same as the classic Kármán vortex street, with vortices being alternately shed from each side of the cylinder.

\subsection{VIV dominated response $\alpha=45^{\circ}$}

Like the case at $\alpha=0^{\circ}$, the case at $\alpha=45^{\circ}$ presents a body that is symmetric with respect to the wake centreline. When the body is fixed, the vortex shedding proceeds quite similarly to the canonical bluff body, the cylinder, resulting in a Kármán vortex 

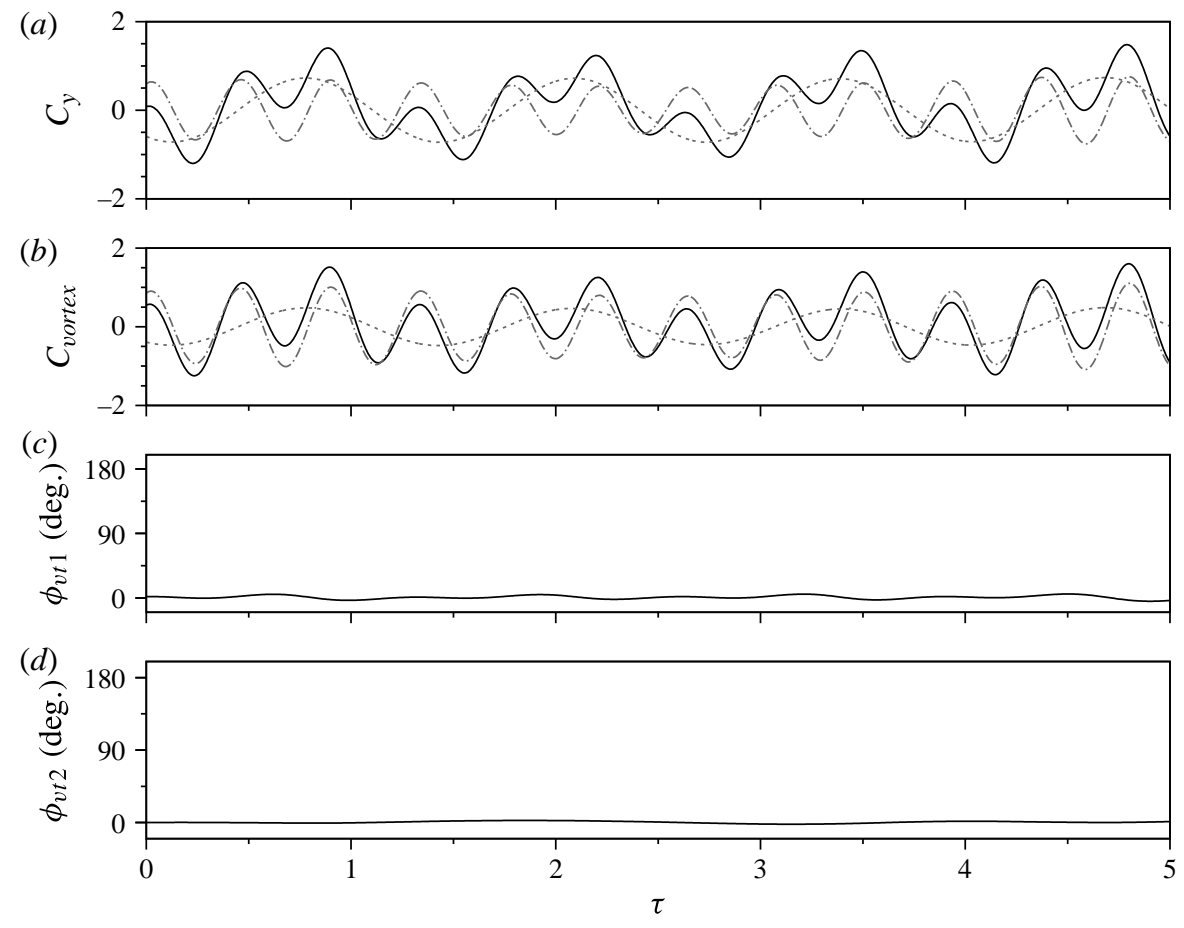

FIgURE 7. Decomposition using the EMD method shows that the first two modes of $C_{y}$ and $C_{\text {vortex }}$ are in-phase in the $1: 3$ synchronization of $\alpha=0^{\circ}$. In $(a)$ and $(b)$, the black solid lines represent $C_{y}$ and $C_{\text {vortex }}$, respectively, in which the grey dashed lines represent the first EMD mode $\left(C_{y(E M D 1)}\right.$ and $\left.C_{\text {vortex }(E M D 1)}\right)$ and the grey dot-dashed lines represent the second EMD mode $\left(C_{y(E M D 2)}\right.$ and $\left.C_{v o r t e x(E M D 2)}\right)$. $(c)$ The relative phase $\left(\phi_{v t 1}\right)$ between $C_{\text {vortex }(E M D 1)}$ and $C_{y(E M D 1)}$, and $(d)$ the relative phase $\left(\phi_{v t 2}\right)$ between $C_{v o r t e x(E M D 2)}$ and $C_{y(E M D 2)}$. The reduced velocity of this case is $U^{*}=14.26$.

street that is spatio-temporally symmetric, i.e. evolution forward in time by half a period plus reflection about the wake centreline returns the original flow (it should be noted that the flow is not exactly spatio-temporally symmetric, due to significant three-dimensional effects, and some fluctuation in the frequency of the shedding over time, but the basic configuration of the vortex shedding does not deviate from this basic spatio-temporal picture significantly).

Unlike the case at $\alpha=0^{\circ}$, the case at $\alpha=45^{\circ}$ is not susceptible to galloping. As explained by the quasi-static theory of Parkinson \& Smith (1964), galloping should only occur when $\partial \overline{C_{y}} / \partial \alpha>0^{\circ}$ at the base angle of attack (here $45^{\circ}$ ). This does occur for $\alpha=0^{\circ}$, but not for $\alpha=45^{\circ}$. However, the $\alpha=45^{\circ}$ case, like any bluff body that causes alternate vortex shedding, is susceptible to VIV, and it is this phenomenon that dominates the FIV of this case.

Nemes et al. (2012) showed that in some respects the response of the square cylinder at $\alpha=45^{\circ}$ is similar to that of the circular cylinder, as described in $\S 2.3$ and shown in figure 3. The primary differences identified were a less distinct transition between the initial and upper branches, and rather than a distinct periodic lower branch that suddenly loses coherence beyond some critical $U^{*}$, a gradual increase in disorder was observed, with significant amplitude oscillations still occurring for the unsynchronized flow. 
(a)

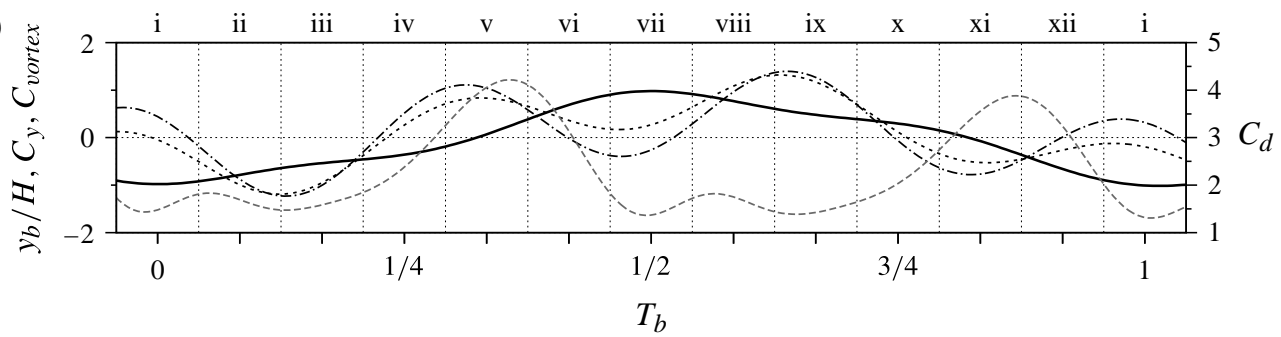

(b)
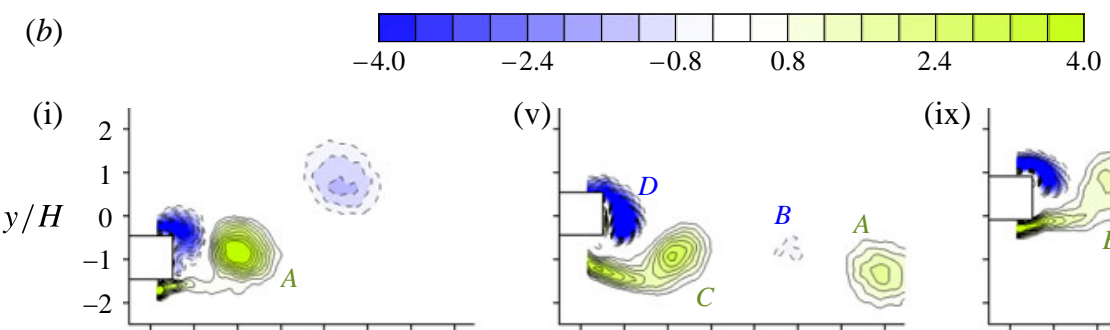

(v)

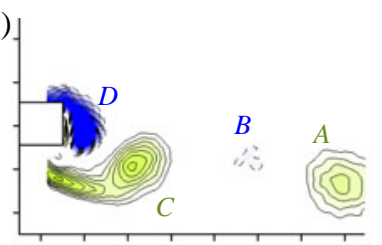

(ix)
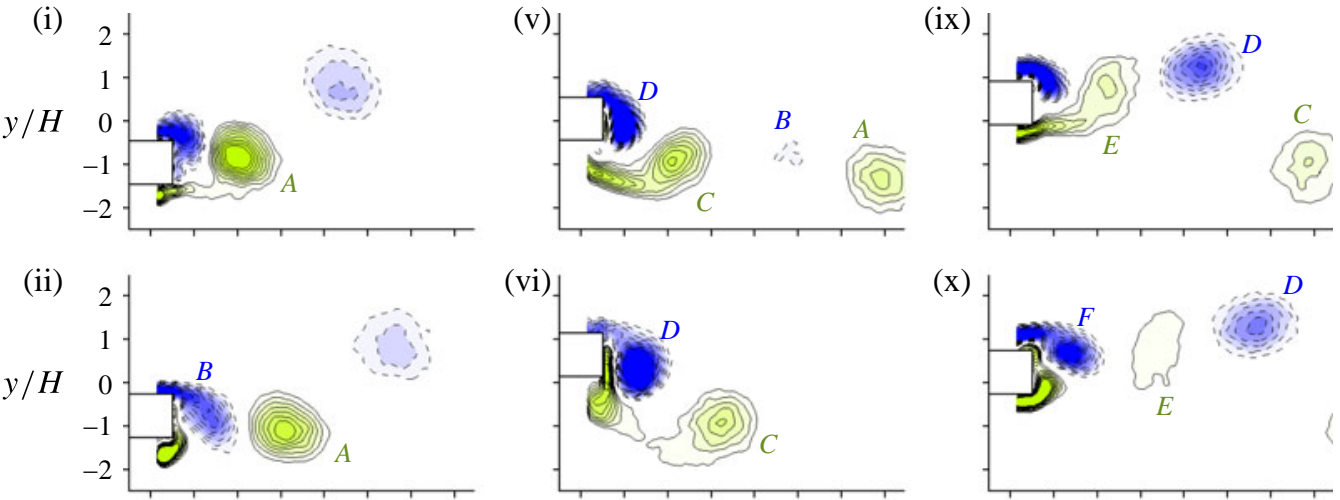

(vi)

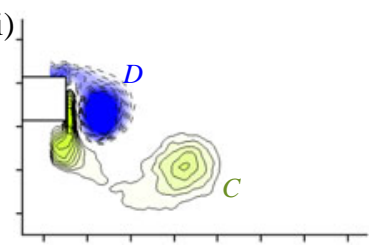

(x)
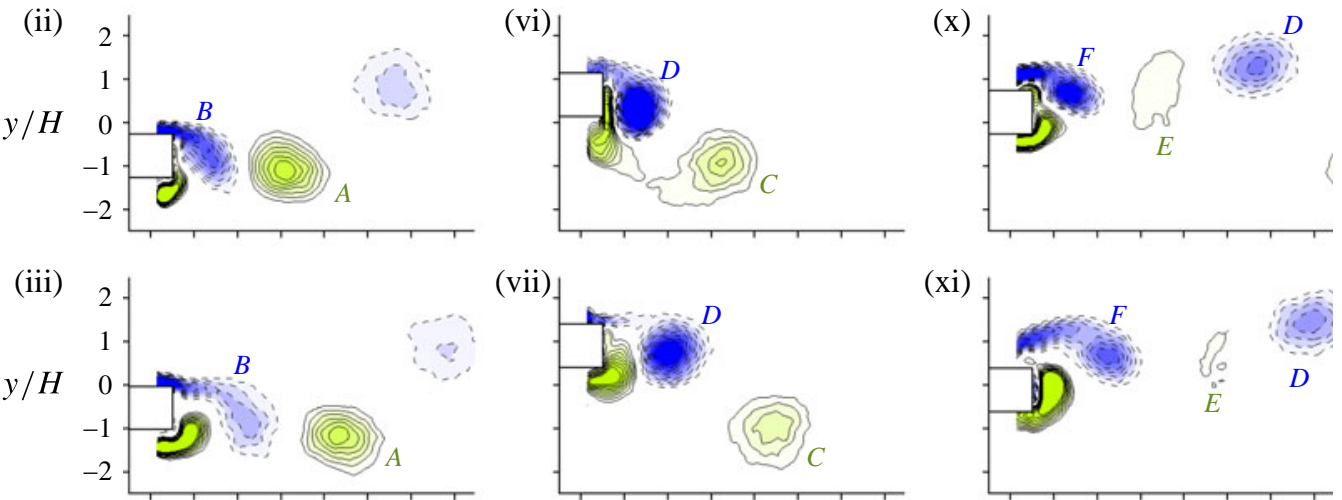

(vii)

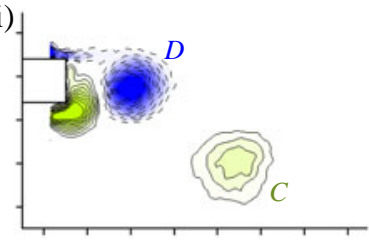

(xi)
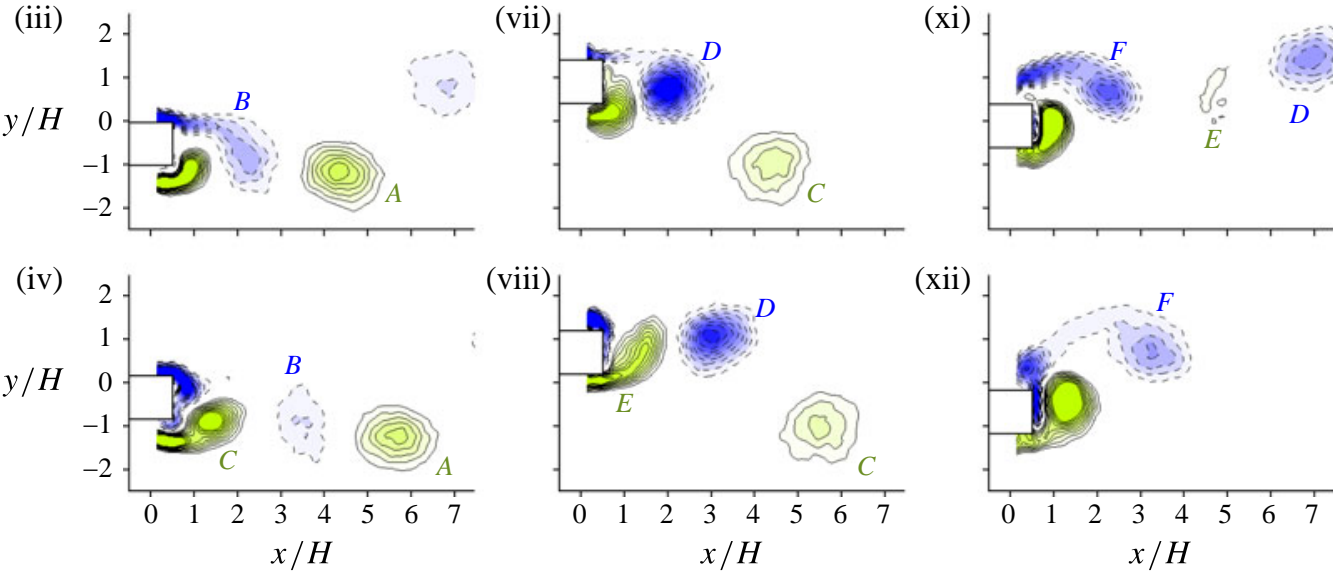

FIGURE 8. (Colour online) Time-based phase-averaged vorticity fields reveal a $3(2 S)$ mode in the 1:3 synchronization regime. Three cycles of the $2 S$ mode are shed in an oscillation cycle. For the case shown, $m^{*}=2.64, \alpha=0^{\circ}$ and $U^{*}=14.26$. (a) The time history of the displacement and forces over a cycle of oscillation. The black solid line represents $y_{b}$, the black short-dashed line represents $C_{y}$, the black dot-dashed line represents $C_{\text {vortex }}$, and the grey long-dashed line represents $C_{d}$. (b) Images of vorticity from PIV averaged over the intervals marked on the time history in $(a)$. The vorticity contour levels shown are normalized by $\omega_{z}^{*}=\omega_{z} H / U$. The contours marked with solid lines represent positive values, and the contours marked with dashed lines represent negative values. 
The results presented here build on and clarify those results. Experiments at very fine increments of $U^{*}$ have been performed in order to home in on the transitions between the various response regimes present. The results presented below show that, in addition to the results presented by previous studies, this upper branch in fact consists of a number of regimes, each with distinct frequency, force, and phase characteristics.

\subsubsection{Amplitude and frequency responses}

Figure $9(a)$ shows the variation of the amplitude of oscillation as a function of $U^{*}$ for a relatively light body at $m^{*}=2.64$, together with the variation of the frequency content of the displacement and force time series (figure $9 b-e$ ). The frequency content plots are constructed in the same manner as figure 5, described in §3.1.1.

Figure 9 shows that the response type is quite varied, with large-amplitude oscillations for $3<U^{*}<7.5$ (the region designated as the upper branch by Nemes et al. 2012) consisting of five regimes, the boundaries of which are marked by the dotted vertical lines on the figure. The characteristics of each of these regimes, as well as the initial branch and desynchronization regions, are highlighted below, in order of increasing $U^{*}$.

For $U^{*}<3$, the flow is on the initial branch. Here, the oscillations are small and modulated, and figure 9 shows that the oscillation as well as the lift force consists of frequency components at both the Strouhal and natural frequencies.

At $U^{*}=3$, the flow transits to the beginning of the upper branch, and the first of a series of synchronized regimes, where the vortex shedding and the body oscillation occur at the same frequency. In this first regime (i), the vortex shedding occurs in the $2 S$ mode, similar to the classic Kármán vortex street. The frequency of oscillation is close to, but slightly below, the natural frequency of the body. The amplitude of oscillation in this regime is almost a linear function of $U^{*}$. The onset of this regime is also marked by a distinct increase in the magnitude of the lift force measured on the body, as shown in figure 10 .

At $U^{*}=4.4$, a change to a second distinct synchronized regime (ii) occurs. The wake mode changes from the $2 S$ mode to the $2 P$ mode, as shown in figure 11 . This is accompanied by a small but distinct kink in the trend of amplitude as a function of $U^{*}$. There is also a distinct change in the frequency response. The frequency of oscillation and vortex shedding shifts to a value close to, but now slightly above, the natural frequency of the body. This appears to be related to a change of the added mass related to the redistribution of vorticity that occurs with the change of wake mode (see Lighthill 1986). Figure 10 shows that with the onset of this regime and the change in wake mode, there is also a switch in the vortex phase from $\phi_{\text {vortex }}=0^{\circ}$ to $\phi_{\text {vortex }}=180^{\circ}$, and a jump in the magnitude of the vortex lift.

At $U^{*}=5$, a third distinct synchronized regime (iii) begins. The onset of this regime occurs close to the point where the body natural frequency is equal to the Strouhal frequency. There is little discernible difference in the flow between this regime and the previous one (ii); however, there is a distinct change in the frequency of response as shown in figure 9 . The frequency moves from a value close to the natural frequency, to a value close to, but slightly above, the Strouhal frequency. The flow remains synchronized; both the vortex shedding and the body oscillation occur at this value close to the Strouhal frequency, and the governing FIV phenomenon appears to be VIV. Regardless, the frequency is not set by the body natural frequency as it invariably is during synchronized VIV of a circular cylinder. 

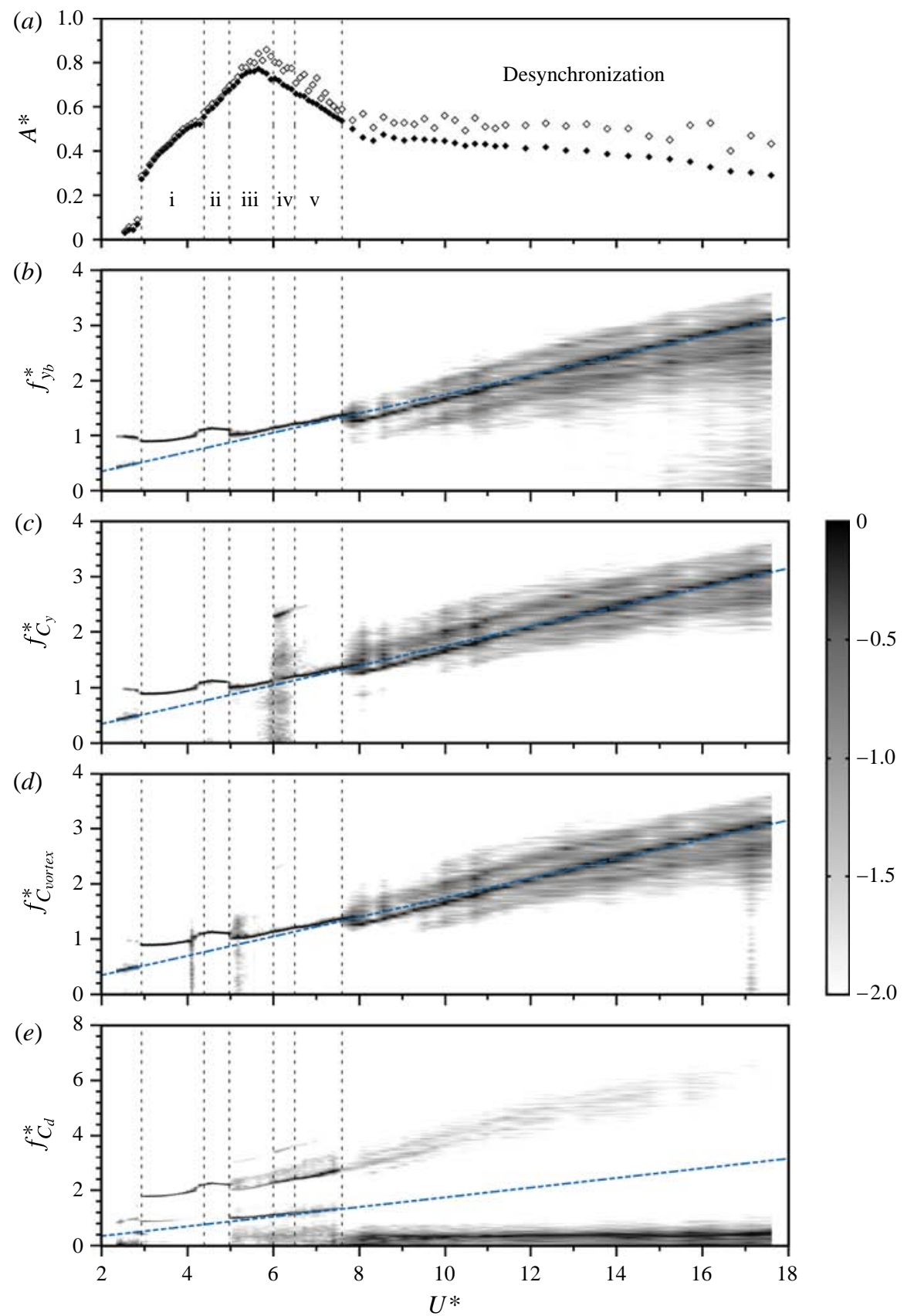

FIGURE 9. (Colour online) Various response regimes (separated by vertical dotted lines, and labelled $\mathrm{i}-\mathrm{v}$ on $(a)$ ) occur as a function of $U^{*}$ for the $\alpha=45^{\circ}$ case, demarcated by their amplitude, frequency, and phase characteristics. In $(a)$, open and solid squares represent $A_{\max }^{*}$ and $A_{10}^{*}$, respectively; $(b)-(e)$ are the logarithmic-scale frequency power spectrum contour plots of the body oscillation $\left(f_{y_{b}}^{*}\right)$, transverse lift $\left(f_{C_{y}}^{*}\right)$, transverse vortex force $\left(f_{C_{\text {vortex }}^{*}}^{*}\right)$, and $\operatorname{drag}\left(f_{C_{d}}^{*}\right)$, respectively. Note that the frequency components are normalized by $f_{N w}$, e.g. $f_{y_{b}}^{*}=f_{y_{b}} / f_{N w}$. The dashed line on $(b)-(e)$ marks the Strouhal frequency. 

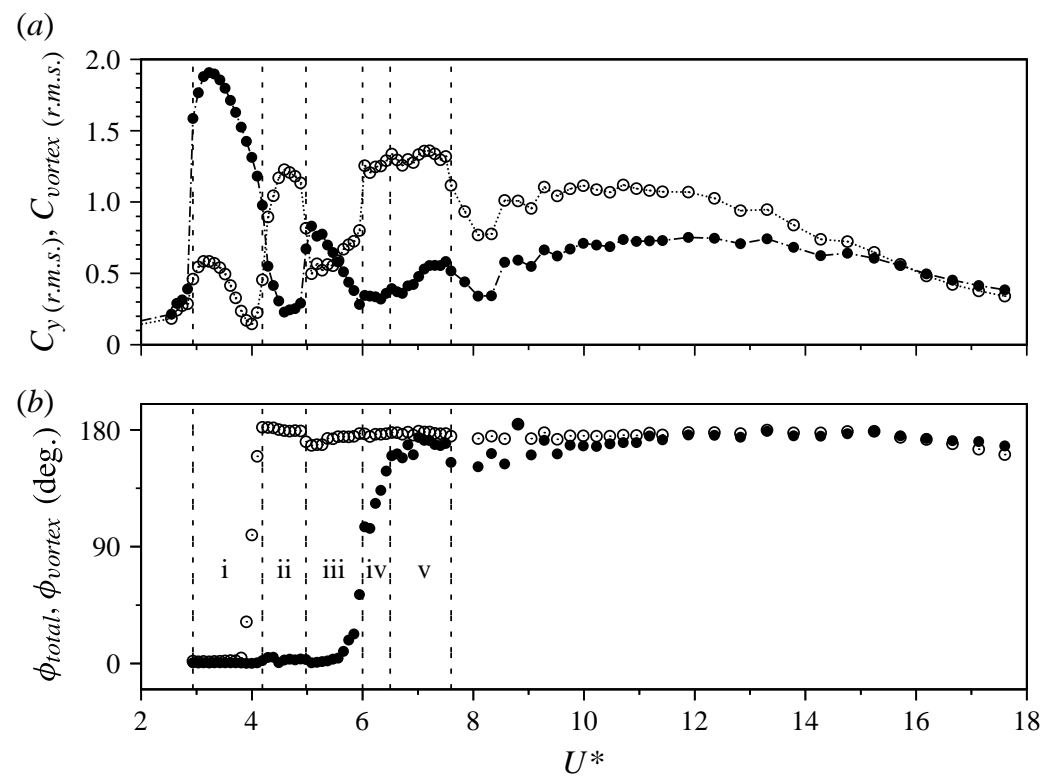

Figure 10. (a) The root mean square (r.m.s.) of the total and vortex lift force and (b) the average phase between the total lift and the body displacement, and the vortex lift and the body displacement. Solid circles represent quantities relating to the total force, and open circles represent quantities relating to the vortex force.

Figure 9 shows that the amplitude of oscillation first increases, then decreases with increasing $U^{*}$ in this regime. Figure 10 shows that the onset of this mode is accompanied by a sharp increase in the total lift force, which then linearly decreases with $U^{*}$. The vortex force, on the other hand, increases with $U^{*}$.

A further interesting feature of this regime is the fact that the drag appears to oscillate at a frequency that is the same as for the lift, rather than a frequency twice that of the lift. A significant component is present at twice the primary frequency of the lift; however, the strongest component is the same as that of the lift. One way for this to occur is for the vortex shedding to be asymmetric, which should result in a small non-zero mean lift and displacement. However, a non-zero mean lift is not immediately apparent in the results of the current experiments. A subtle asymmetry may exist that is the cause of the spectral characteristics of the drag, but further experiments are required to fully understand this feature.

The reason why a non-zero mean displacement is difficult to measure is that the exact equilibrium position of the body in the channel is unknown. The resting position of the body while there is no flow in the channel could be used, but this assumes that the inflow is perfectly aligned. The error in the actual resting position from making this assumption is small, but it may be of the same order as the mean displacement for a flow that is only weakly asymmetric. Strong asymmetries will be clear from the shape of the time history, i.e. for a periodic flow, $y(t+T / 2) \neq-y(t)$. However, for a weakly asymmetric flow, it is difficult to separate such inherent asymmetries from fluctuations in the body position due to external disturbances.

$U^{*}=6$ marks the beginning of a fourth regime (iv), distinct from the others in the high-amplitude upper branch region as it is not periodic. Figure 9 shows that while the frequency content of the body oscillation remains dominated by a single component at 

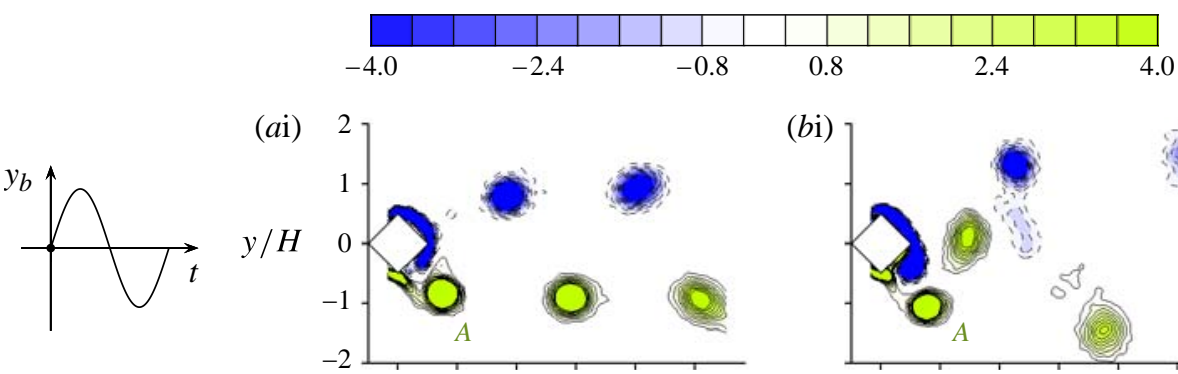

(bi)
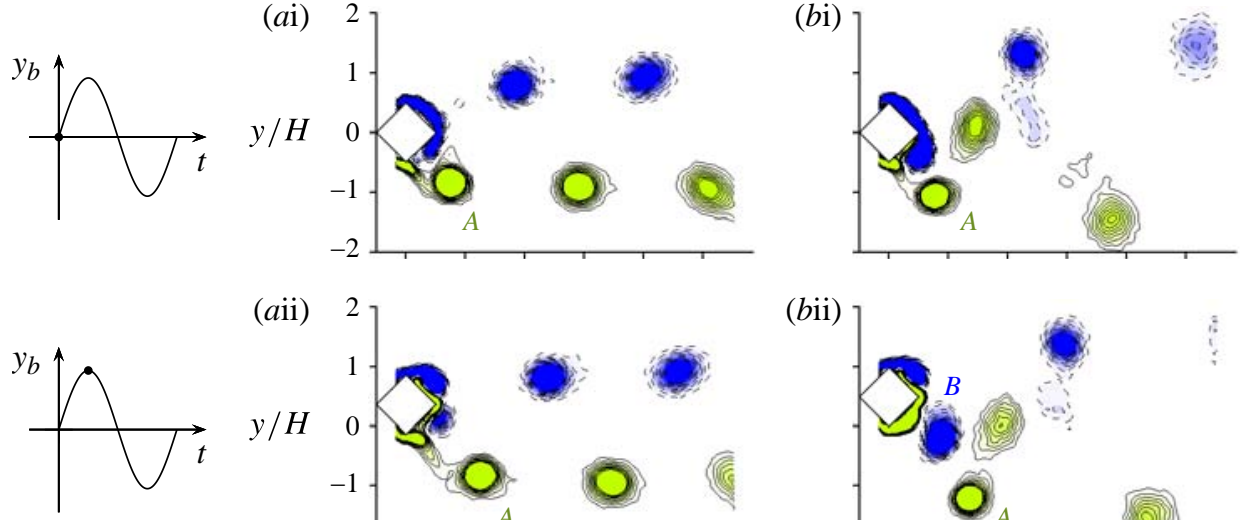

(aii)

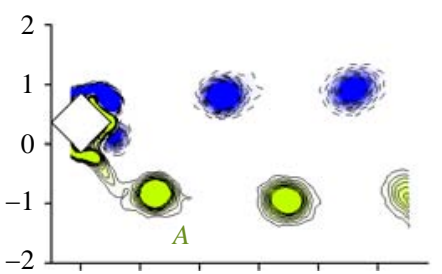

(bii)
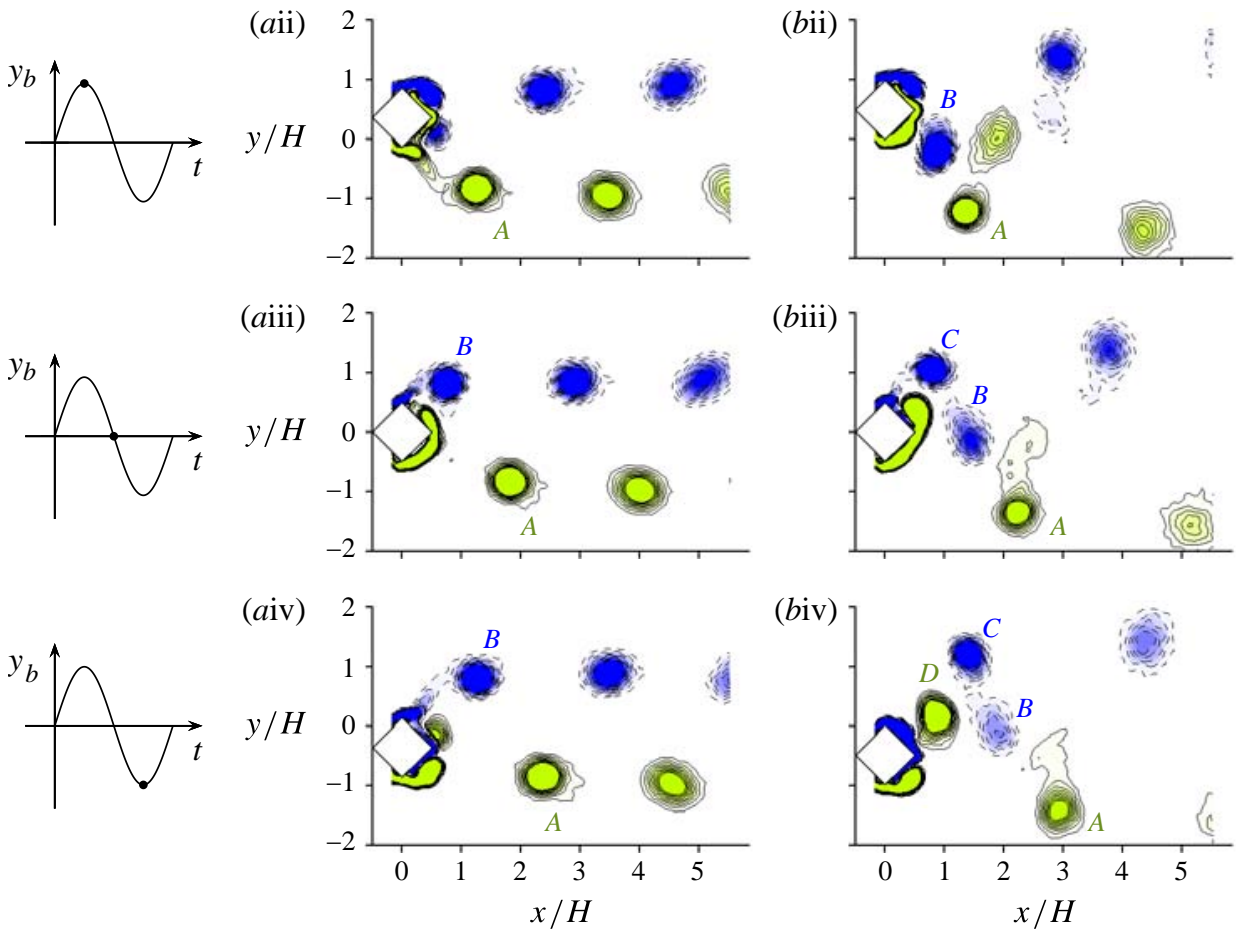

FIGURE 11. (Colour online) Phase-averaged vorticity fields show the $2 S \leftrightarrow 2 P$ wake mode transition. The schematics on the left show the position in the oscillation cycle of the corresponding images. (a) The different phases of the $2 S$ mode at $U^{*}=3.28$; $(b)$ the different phases of the $2 P$ mode at $U^{*}=4.29$. Note that the vorticity contour levels shown are normalized by $\omega_{z}^{*}=\omega_{z} H / U$. The contours marked with solid lines represent positive values, and the contours marked with dashed lines represent negative values.

a value slightly above the Strouhal frequency, the lift force shows a more broadband response, focused around harmonics of the oscillation frequency. This is indicative of a vortex shedding process that is 'almost' periodic.

It is possible that this regime is not inherent, but is rather a response of the system to the external noise in any experimental setup. Figure 10 shows that the onset of this regime is also the point where the total phase switches from $\phi_{\text {total }}=0^{\circ}$ to $\phi_{\text {total }}=180^{\circ}$. Along with this, the figure shows that the total lift force is close to the minimum recorded. This is consistent, as the shift in $\phi_{\text {total }}$ is most likely driven by a change in the balance of two opposing forces, as explained by Carberry et al. (2005). This fine balance of forces may render the system more susceptible to noise 

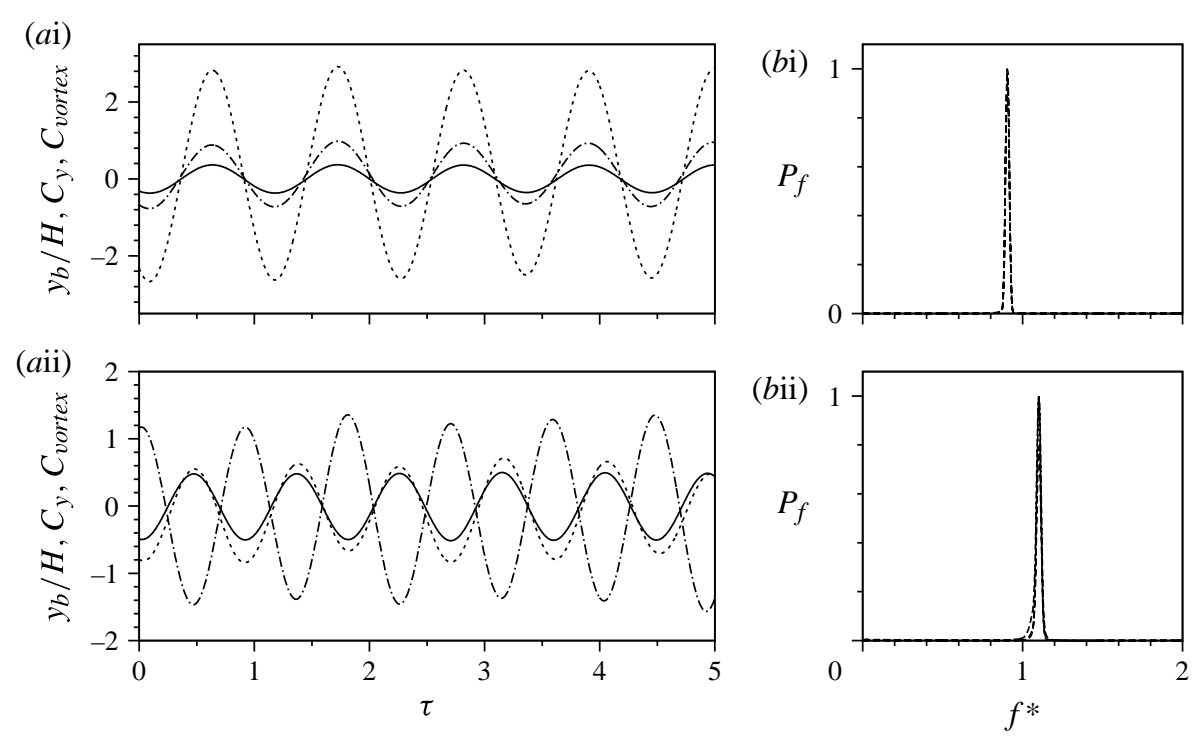

FIGURE 12. A jump of $0^{\circ} \leftrightarrow 180^{\circ}$ in the vortex phase, and switch in the frequency from slightly below to slightly above the natural frequency, which are associated with the $2 S \leftrightarrow$ $2 P$ wake mode transition, as the flow changes from regime (i) to regime (ii) for the $\alpha=$ $45^{\circ}$ case. Time traces of $(a)$ the displacement (solid lines), transverse lift (dashed lines) and vortex force (dot-dashed lines), and $(b)$ power spectra (normalized by the peak values) for cases $\alpha=45^{\circ}, m^{*}=2.64$ at (i) $U^{*}=3.28$ and (ii) $U^{*}=4.29$.

than normal. Further evidence for this regime being a function of external forcing is that the body oscillation frequency and amplitude are practically continuous across regimes (iii), (iv), and (v), indicating there is little change in the wake configuration across these regimes.

Regime (v) begins at $U^{*}=6.5$ and continues to $U^{*}=7.5$ where the body oscillation and vortex shedding become desynchronized. Like regime (iii), regime (v) consists of synchronized oscillations at a frequency slightly above the Strouhal frequency. Also like regime (iii), the drag has a strong component at the same frequency as the lift and the displacement; however the strongest component is at a frequency twice this value. Any asymmetry present in regime (iii) may therefore still occur here.

Finally, at $U^{*}=7.5$, the body oscillation and the vortex shedding become desynchronized. The vortex shedding occurs at a primary frequency close to, but slightly below, the Strouhal frequency for the fixed body; however the spectra are quite broadband indicating that the flow is not strictly periodic. The amplitude of oscillation is only a weak negative function of $U^{*}$ as shown in figure 9 , as is the lift force as shown in figure 10.

\subsubsection{Further details of the $2 S \leftrightarrow 2 P$ wake mode transition}

Figure 12 shows time traces of the oscillation and forces and frequency spectra for two cases; one at $U^{*}=3.28$, in regime (i), and one at $U^{*}=4.29$, in regime (ii). The periodicity of the oscillation in both regimes is clear from the time traces. The switch in $\phi_{\text {vortex }}$ that occurs is also clear. Comparison of the spectra of the two cases also shows very explicitly the switch from a frequency just below the natural frequency, to a frequency just above the natural frequency. 
Figure 11 above shows sequences of images of the wake, visualized using vorticity, from phase-averaged PIV measurements. From left to right, the figure shows a column of schematics indicating the point in the oscillation cycle at which each image is taken, then a column of images of the wake in regime (i) at $U^{*}=3.28$, then a column of images of the wake in regime (ii) at $U^{*}=4.29$.

The change in wake mode between the two cases is clear. Regime (i) displays a $2 S$ mode, with alternating vortex shedding from each side of the body. Regime (ii) displays a $2 P$ mode, where during one half-cycle a positive vortex shed from the bottom edge of the body is paired with a negative vortex shed from the rear edge of the body. The inverse occurs in the following half-cycle, with a negative vortex shed from the top edge paired with a positive vortex shed from the rear edge.

\subsection{Modified VIV response $\alpha=20^{\circ}$}

While the case at $\alpha=0^{\circ}$ is clearly dominated by the phenomenon of galloping, and the case at $\alpha=45^{\circ}$ is dominated by the phenomenon of VIV, the governing phenomenon for the case at a resting angle of attack of $\alpha=20^{\circ}$ is not as clear. The complicated regime map presented in Nemes et al. (2012) shows that there is at least one new response regime present, labelled the 'higher' branch, as it consists of well-organized oscillations at amplitudes larger than those present in the upper branch.

This section presents results of experiments at $\alpha=20^{\circ}$ at very fine increments of $U^{*}$, so that the extent of the response regimes identified can be clearly identified. The results show that there is indeed an upper and a higher branch of response, separated on the $U^{*}$ axis by a region of chaos that appears to be driven by intermittency between the two modes. In addition, the results presented here show the higher branch is a type of subharmonic response, with two vortex shedding cycles occurring over one body oscillation cycle. The vortex formation and shedding process, which for this branch depends on vorticity created at different faces of the body, is also presented.

\subsubsection{Amplitude and frequency responses}

Figure 13 shows the amplitude of the body oscillation as a function of $U^{*}$, as well as the frequency content of the displacement and various forces, for the $\alpha=20^{\circ}$ case. Again, the plots are constructed in the same manner as those of figure 5 as explained in $\$ 3.1 .1$.

Observation of the amplitude response in figure 13(a) clearly shows four response regimes: an initial branch (IB), an upper branch (UB), a higher branch (HB), and a desynchronization region where the amplitude is approximately constant. Both the upper and higher branches consist of ordered, periodic oscillations, hence both are highlighted with dark shading in the figure. Outside of these two regimes, the flow response can be more complicated. These various response regimes are outlined below.

The initial branch consists of small-amplitude oscillations. The oscillations are modulated, as both the Strouhal frequency (the vortex shedding frequency from the rigid body) and the body natural frequency play a role. This behaviour is similar to the initial branch that occurs in VIV studies of a circular cylinder.

Increasing $U^{*}$ beyond $U^{*}=4$ sees the flow transit to the upper branch. With the onset of the upper branch, the oscillations become regular and periodic with amplitude increasing with $U^{*}$. Like the classic upper branch identified in flows past a circular cylinder, here the frequency appears to lock on to a frequency close to the natural frequency. This is clearly evident in the plots of frequency content shown in figure $13(b-e)$, that show only a single line for the displacement and the lift 

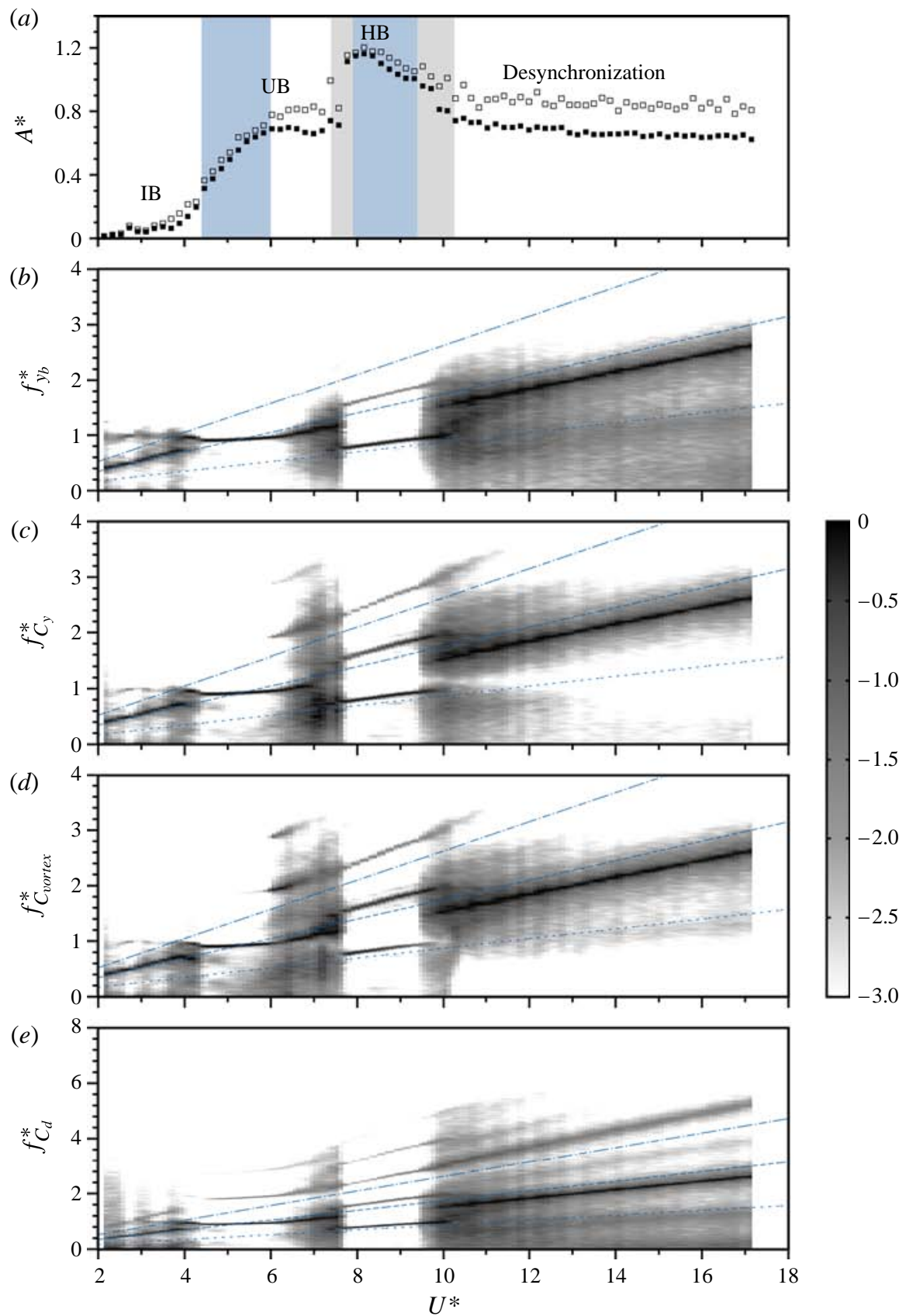

FIgURE 13. (Colour online) The amplitude $(a)$ and frequency response $(b)-(e)$ as a function of $U^{*}$ with $m^{*}=2.64$ at $\alpha=20^{\circ}$, showing two resonance regions shaded in dark grey in $(a)$. In $(a)$, open and solid squares represent $A_{\max }^{*}$ and $A_{10}^{*}$, respectively; logarithmic-scale frequency power spectrum contour plots of $f_{y_{b}}^{*}, f_{C_{y}}^{*}$ and $f_{C_{\text {vortex }}^{*}}^{*}$ in $(b)-(d)$ clearly show that in the upper branch (UB) the body oscillation synchronizes with the natural frequency, and then to half of the Strouhal frequency in the higher branch (HB). The higher branch is bounded by intermittent regions (shaded in light grey) on either side. Note that the frequency components are normalized by $f_{N w}$, e.g. $f_{y_{b}}^{*}=f_{y_{b}} / f_{N w}$. The slope values of the dotted, dashed, and dot-dashed lines represent $S t / 2$, St and $2 S t$, respectively. 
force that remains close to the natural frequency. The range of $U^{*}$ over which this synchronization occurs is below the value of $U^{*}$ at with the natural frequency is equal to the Strouhal frequency, ending when $U^{*}$ is such that the Strouhal frequency is approximately equal to the natural frequency.

At the point where the synchronization of the upper branch is lost, the flow becomes chaotic, with a broadband frequency response focused around a frequency slightly below the Strouhal frequency. The amplitude of oscillation is approximately constant with further increases in $U^{*}$. Here, the flow is desynchronized, and this regime is similar to the desynchronized regime found at high $U^{*}$ for the $\alpha=45^{\circ}$ case.

Unlike the $\alpha=45^{\circ}$ case, this desynchronization region does not then persist for all higher $U^{*}$. Instead, just prior to $U^{*}=8$, the flow reorganizes and the higher branch regime begins, as first identified by Nemes et al. (2012), where it was hypothesized that this regime was a type of interaction mode between VIV and transverse galloping, based on the high amplitude of the response. However, the high fidelity of the data presented in figure 13 shows that this mode is in fact a type of subharmonic VIV. The frequency content of the displacement shown in figure 13 shows that throughout the higher branch regime, the flow is very well-organized and periodic, with a single dominant frequency, which remains very close to half of the Strouhal frequency (in fact just slightly above it). The same frequency appears in the lift, along with a significant component at twice this frequency, which becomes the dominant frequency in the vortex lift. This indicates that this mode consists of two cycles of vortex shedding per oscillation cycle, which is further discussed in $\$ 3.3 .3$.

The onset of the higher branch occurs intermittently with increases in $U^{*}$. From around $U^{*}=7.5$, small periods of organized flow appear intermingled with periods of disorder. These periods of organized flow persist longer and occur more rapidly as $U^{*}$ is increased, before the higher branch becomes 'locked' around $U^{*}=8$. This behaviour indicates that this transition occurs through type-one intermittency, as described by Pomeau \& Manneville (1980). A similar process occurs at the other end of the range of existence of the higher branch. Increasing $U^{*}$ beyond around $U^{*}=9.5$ sees the organized flow interrupted by small periods of disorder, which become longer and more prevalent at $U^{*}$ is further increased, until no evidence of the higher branch remains beyond $U^{*}=10.5$.

Further increases in $U^{*}$ see no further qualitative changes in the flow. The frequency response becomes quite broadband, though focused around a frequency slightly below the Strouhal frequency. The vortex formation and shedding are desynchronized from the oscillation, even so the amplitude remains quite high. Qualitatively, the flow is similar to the desynchronized region that divides the upper and higher branches.

Figure 13 gives a good overview of the flow regimes present for the case at $\alpha=20^{\circ}$. It shows that there are a number of regimes present, all with unique characteristics. Further details of each of these flow regimes are presented in the following sections.

\subsubsection{The upper branch}

The upper branch is characterized by oscillation and vortex shedding close to the Strouhal frequency. Figure 14 shows time traces and frequency spectra of the displacement and forces for a representative case at $U^{*}=5.1$. The oscillations are close to periodic, as is the lift force, and only a single predominant frequency is present in the spectra.

Figure 15 shows twelve images of the wake, visualized as contours of vorticity, over one oscillation cycle. The images were obtained by averaging a number of PIV 

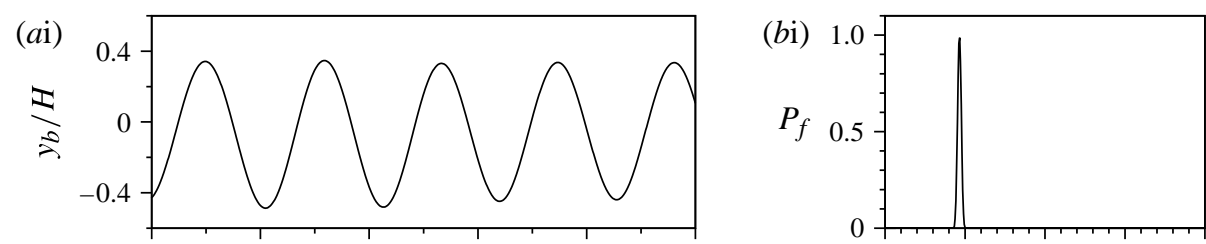

(aii)

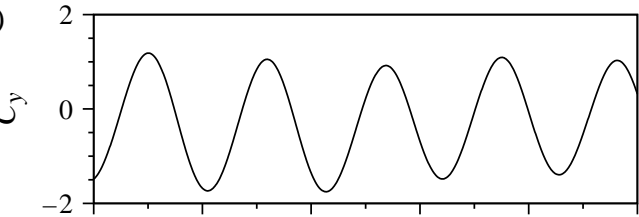

(bii)
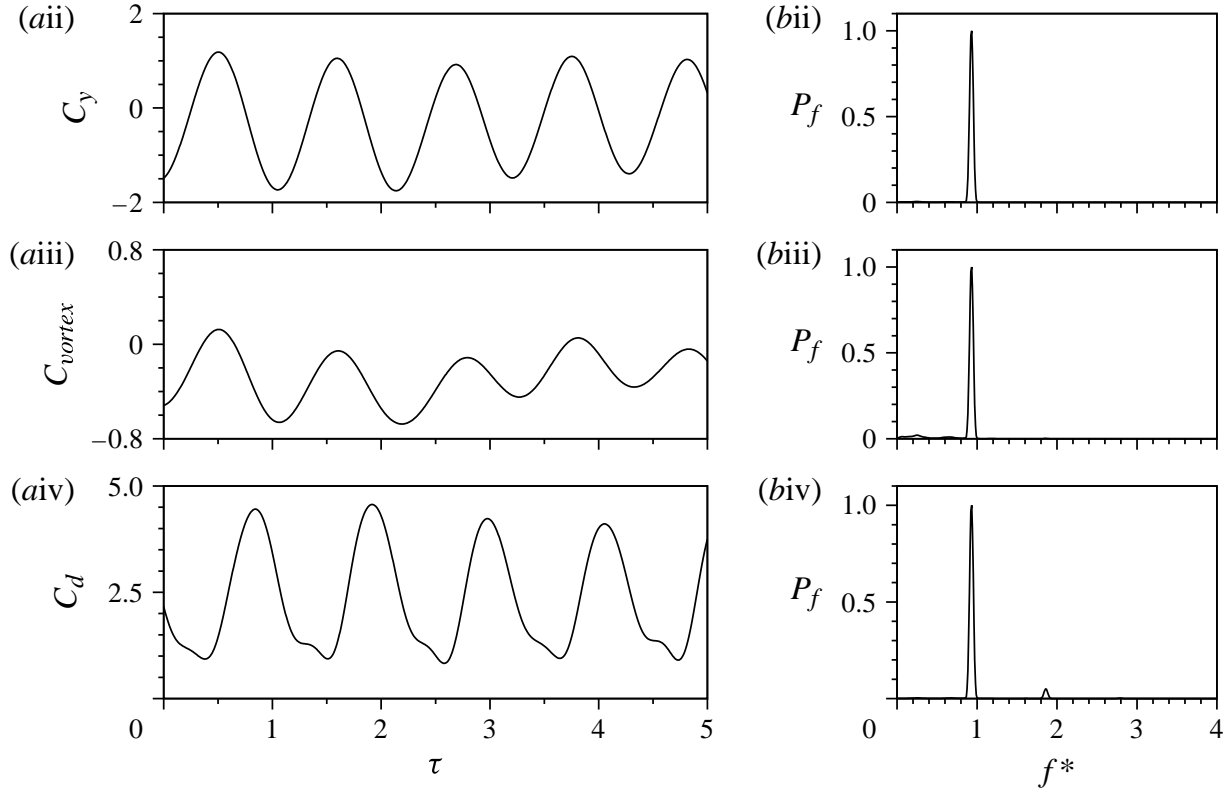

FIgURE 14. Time histories for the case at $\alpha=20^{\circ}$ and $U^{*}=5.1$, in the upper branch. (a) Time traces, and (b) normalized power spectra, of (i) displacement, (ii) total lift, (iii) vortex lift, and (iv) drag.

images over a short time span. The time span for the averaging used for each period is marked on the time history shown in figure $15(a)$.

One feature of note in figure 15 is that even though the total lift and the vortex lift force are approximately sinusoidal, the timing of the vortex shedding is not the same on either side of the wake. This is clear when the streamwise spacing between consecutive vortices is compared. For instance, in the first image moving downstream from left to right, the streamwise distance from the first positive (solid line) vortex on the bottom side to the first negative (dashed line) vortex on the top side is much less than the distance from that first negative vortex to the second positive vortex. This is perhaps not surprising considering the asymmetry of the body.

The formation of the negative vortex on the top side of the body should also be noted. This formation occurs primarily during the second half (the downstroke) of the oscillation cycle, starting from image (vii) in the sequence shown in figure 15 . These images show that initially nascent vortices form, one from the leading edge and one from the trailing edge of the body. It is hypothesized that the trailing-edge vortex is actually formed during the upstroke of the body on the back face, as evidenced from images (i)-(v) of the sequence. Even though it is formed, this trailing-edge vortex is not shed into the wake. Rather, it is eventually amalgamated into the vortex that is formed from the leading edge as shown in images (vii)-(x), and this final single vortex is shed into the wake. This complex vorticity production and amalgamation may go 
(a)

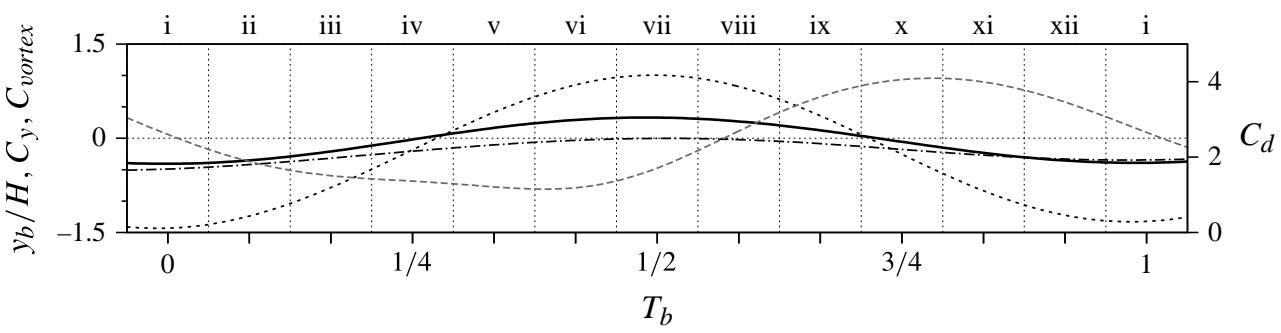

(b)

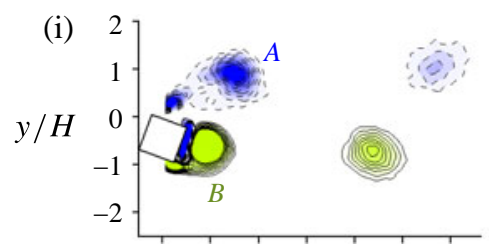

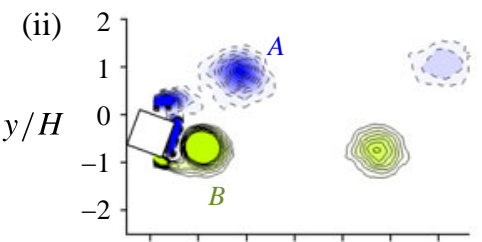

(vi)
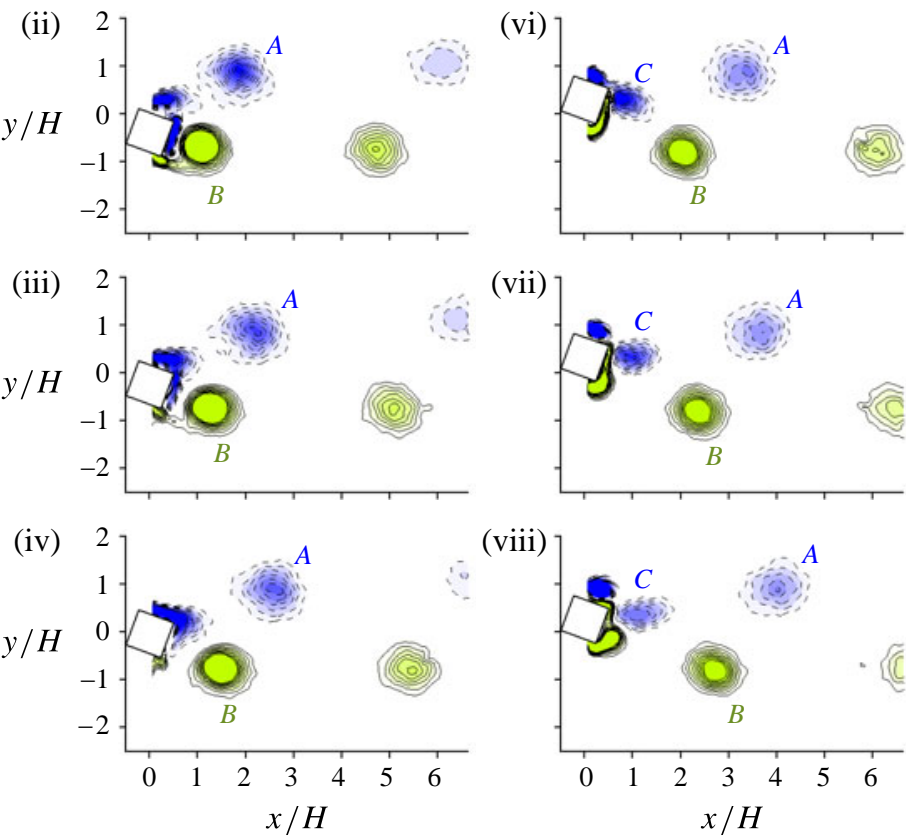

(vii)

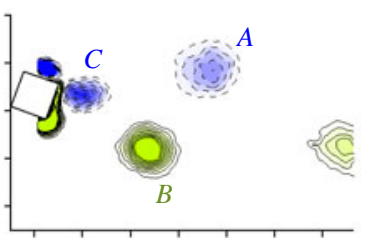

(viii)

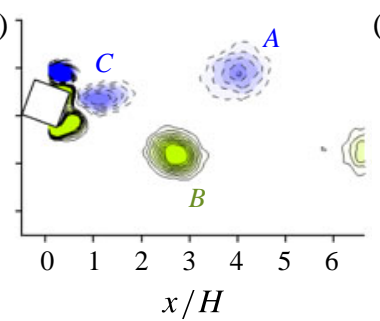

(v)

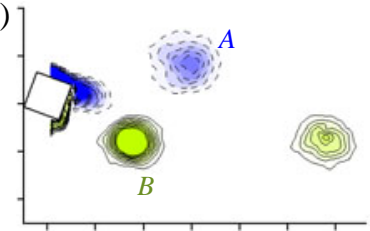

(x)

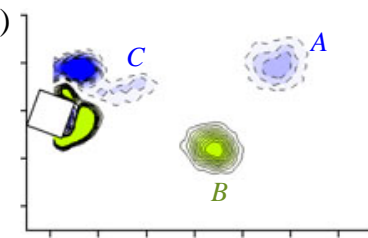

(xi)

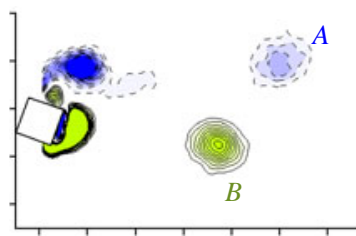

(xii)

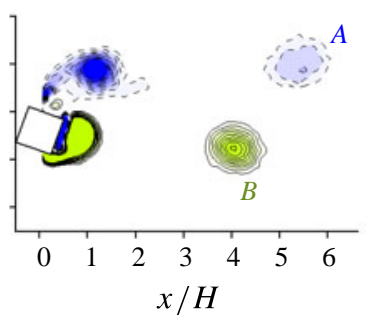

FIGURE 15. (Colour online) Time-based phase-averaged vorticity fields in the upper branch, for the case $m^{*}=2.64$ at $\alpha=20^{\circ}$ and $U^{*}=5.1$. (a) The time history of the displacement and forces over a cycle of oscillation. The black solid line represents $y_{b}$, the black short-dashed line represents $C_{y}$, the black dot-dashed line represents $C_{v o r t e x}$, and the grey long-dashed line represents $C_{d}$. $(b)$ Images of vorticity from PIV averaged over the intervals marked on the time history. The vorticity contour levels shown are normalized by $\omega_{z}^{*}=\omega_{z} H / U$. The contours marked with solid lines represent positive values, and the contours marked with dashed lines represent negative values. The trailing-edge vortex formed during the upstroke is amalgamated with the leading-edge vortex before shedding.

some way to explain the difference in timing of vortex shedding from the top and bottom sides of the body. 

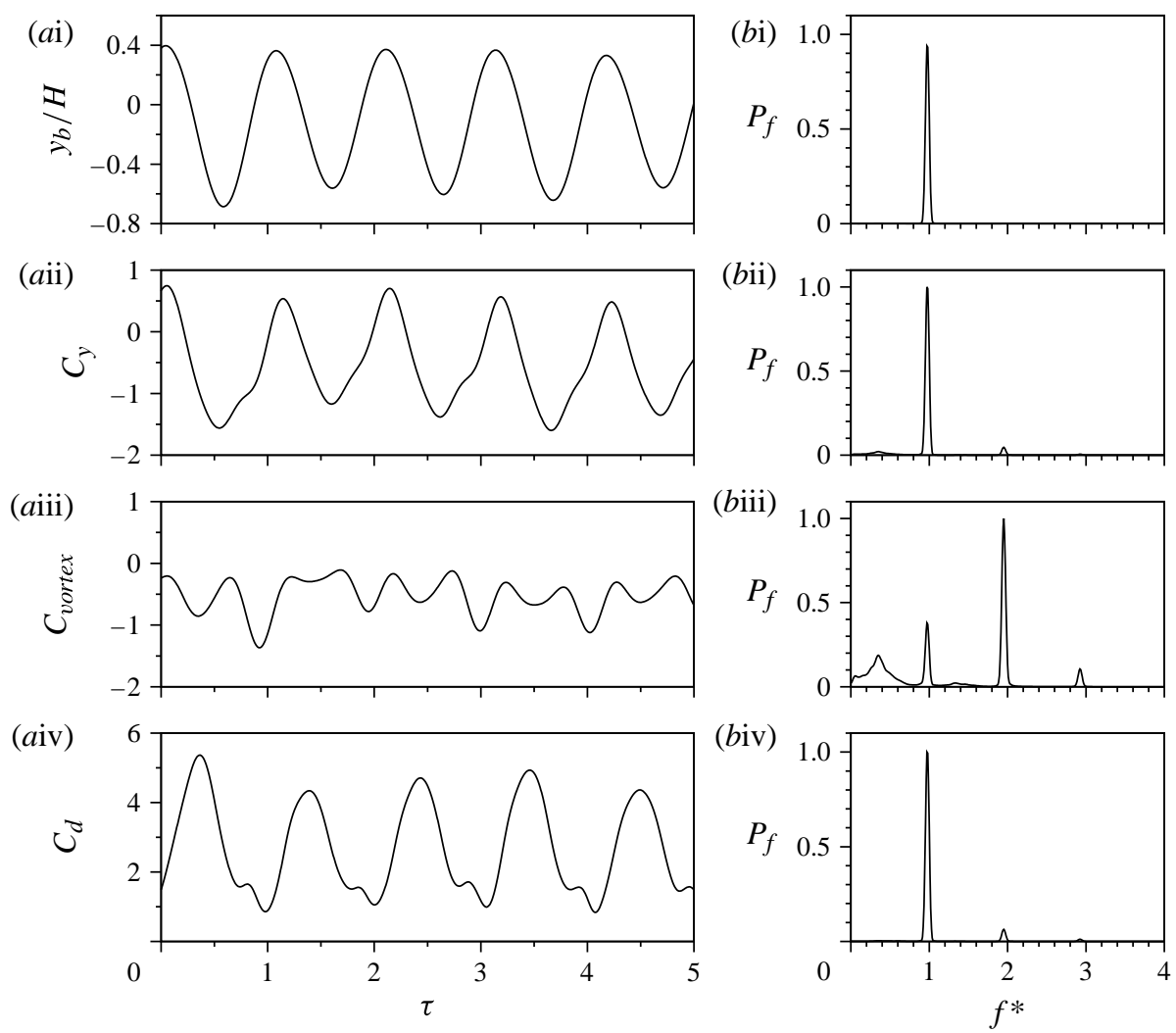

FIgURE 16. (a) Time traces, and (b) normalized power spectra, of (i) displacement, (ii) total lift, (iii) vortex lift, and (iv) drag for the case at $\alpha=20^{\circ}$ and $U^{*}=6.1$.

The delicate vortex formation and shedding process is further highlighted by observing a case that is very close to the upper limit of $U^{*}$ for the upper branch. Figure 16 shows time histories and spectra for such a case at $U^{*}=6.1$. Some similarities with the case at $U^{*}=5.1$ can be found: the primary frequency of oscillation and the total lift force is close to the Strouhal frequency, and the oscillation remains reasonably regular and close to sinusoidal. However, there is a large difference in the vortex lift. While there is still significant energy at the Strouhal frequency, the most energetic component is actually at twice this value, indicating that there has been some kind of change in the vortex formation or timing of vortex shedding.

This change can be seen in the sequence of images of the wake for the $U^{*}=$ 6.1 case, shown in figure 17. Again, the images are of vorticity contours formed by averaging the PIV images over a short time period, which is marked for each image on the time history in figure $17(a)$.

Overall, the shedding process is very similar to the upper branch case at $U^{*}=5.1$ shown in figure 15. However, here, the negative trailing-edge vortex that is formed from vorticity produced on the back face of the body during the upstroke of the body is not simply amalgamated with the vortex forming from the vorticity on the upper side. Instead, this initial trailing-edge vortex is shed into the wake, as shown in images (vi)-(ix) in the sequence of figure 17. However, this vortex is quite weak, and images (x)-(xii) indicate that it quickly dissipates (possibly due to three-dimensional effects) as it travels away from the body. This complex formation-shedding-dissipation cycle 
(a)

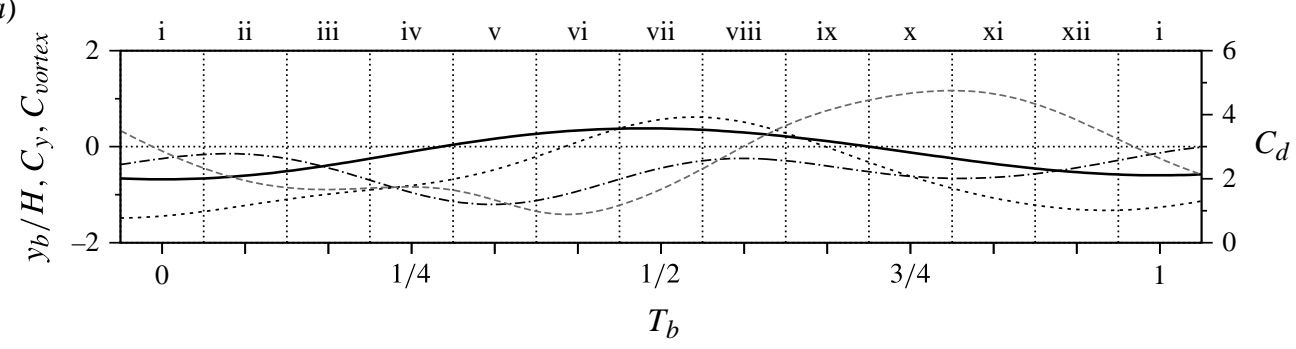

(b)
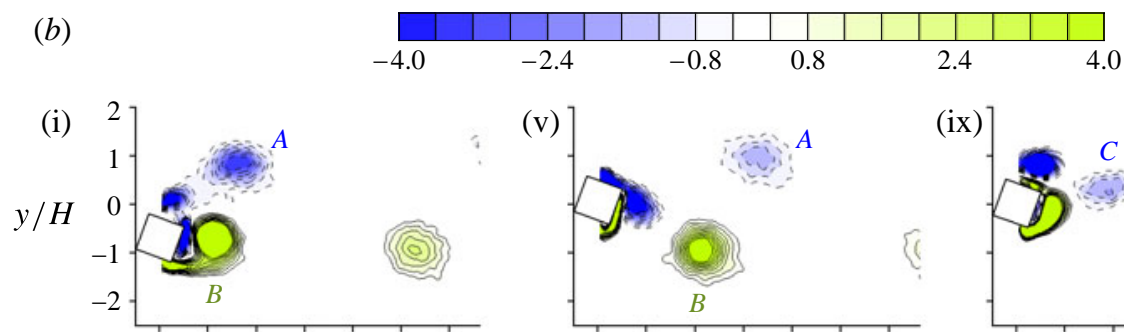

(v)
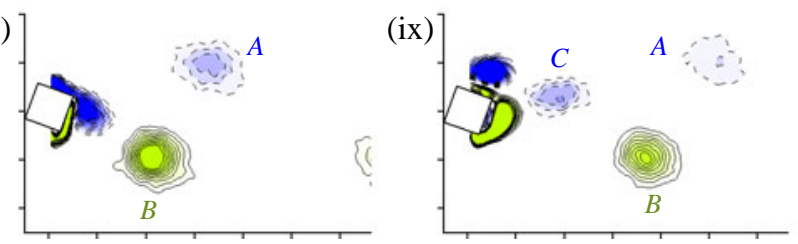

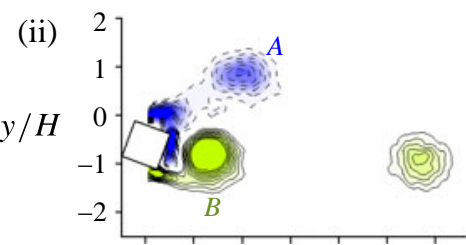

(vi)
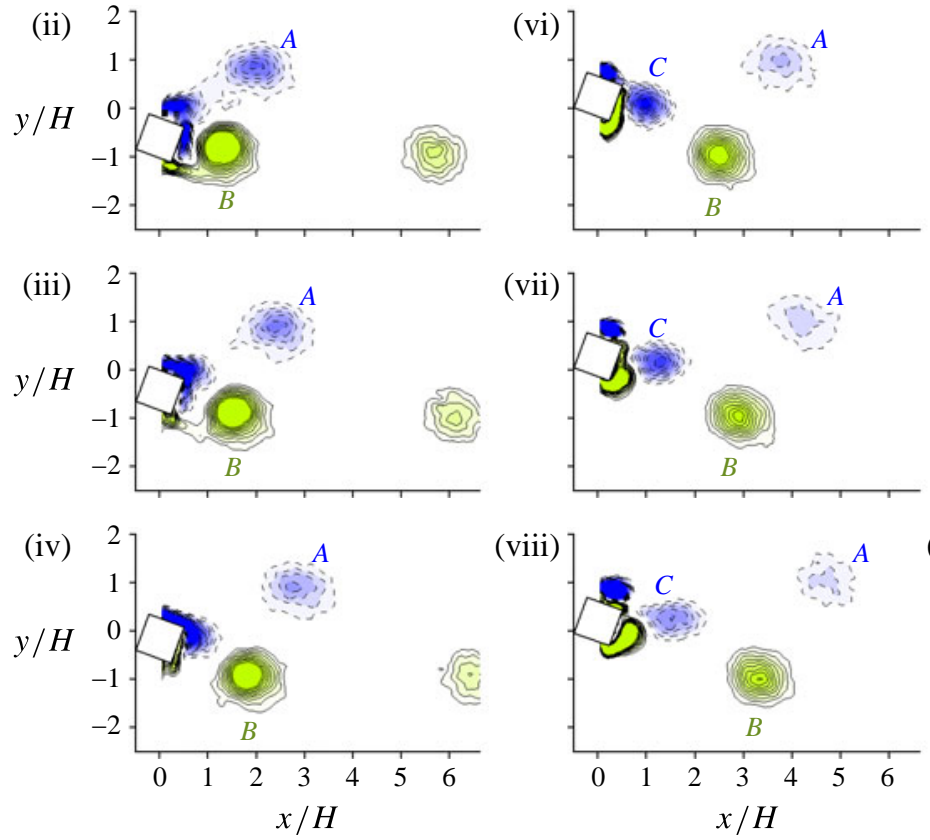

(vii)

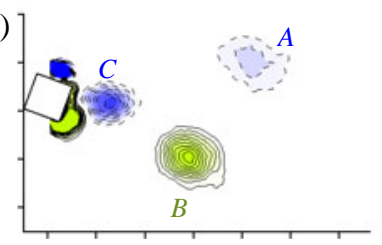

(viii)

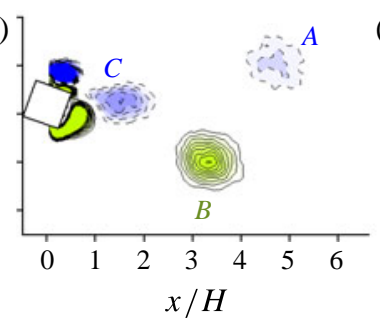

(x)

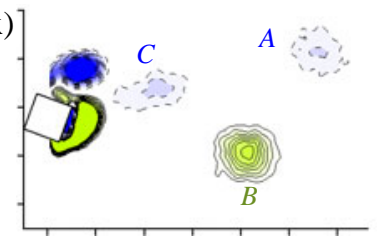

(xi)

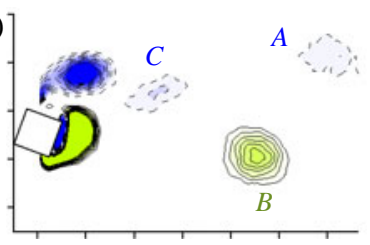

(xii)

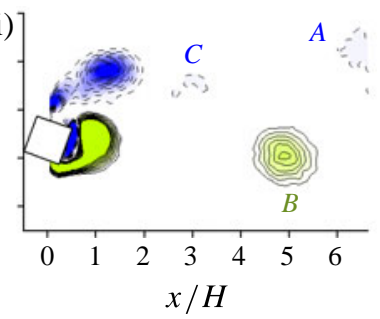

FIGURE 17. (Colour online) Time-based phase-averaged vorticity fields just beyond the upper branch. For this case, $m^{*}=2.64, \alpha=20^{\circ}$ and $U^{*}=6.1$. (a) The time history of the displacement and forces over a cycle of oscillation. The black solid line represents $y_{b}$, the black short-dashed line represents $C_{y}$, the black dot-dashed line represents $C_{\text {vortex }}$, and the grey long-dashed line represents $C_{d}$. $(b)$ Images of vorticity from PIV averaged over the intervals marked on the time history. The vorticity contour levels shown are normalized by $\omega_{z}^{*}=\omega_{z} H / U$. The contours marked with solid lines represent positive values, and the contours marked with dashed lines represent negative values. The trailing-edge vortex formed during the upstroke is shed before the leading-edge vortex, but is quickly dissipated. 

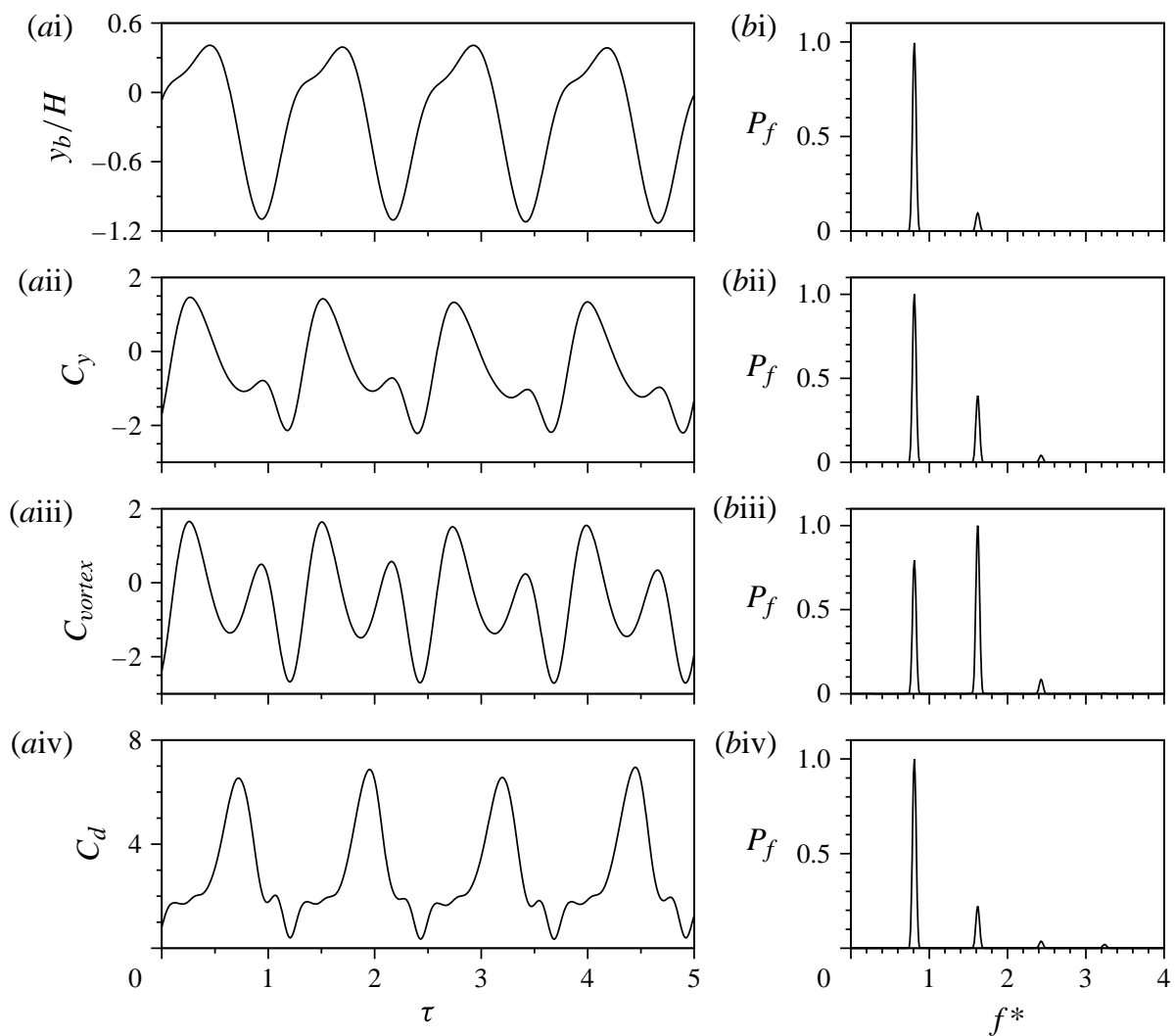

FIgURE 18. Time histories for the case at $\alpha=20^{\circ}$ and $U^{*}=8$, in the higher branch. (a) Time traces, and (b) normalized power spectra, of (i) displacement, (ii) total lift, (iii) vortex lift, and (iv) drag. The subharmonic synchronization between the vortex shedding and body motion is shown by the fact that the primary frequency of the vortex lift is twice that of the body motion.

goes some way to explaining why the synchronization between the body motion and the vortex shedding is lost as $U^{*}$ is increased beyond this point.

\subsubsection{The higher branch}

The higher branch is characterized by oscillation at close to half of the Strouhal frequency. The time trace of the oscillation for a representative case at $U^{*}=8$ is shown in figure 18(ai). The asymmetry of the flow induced by the angle of attack is clear in this figure. Unlike the upper branch, the vortex shedding in the higher branch does not occur at the same frequency as the oscillation. Instead, it occurs at twice the frequency of the oscillation. The influence of this higher harmonic is clear in the time trace of the total lift force shown in figure 18(aii), and the presence of significant energy at twice the oscillation frequency is shown in the corresponding spectrum of figure 18(bii). This effect is even more pronounced in the vortex lift, where the energy in the spectrum at twice the oscillation frequency is greater than the energy at the oscillation frequency.

This subharmonic synchronization between the oscillation and the vortex shedding, where the oscillation is synchronized to half the vortex shedding frequency, is clearly shown in the images of figure 19. Here is shown a sequence of twelve images of the 
(a)

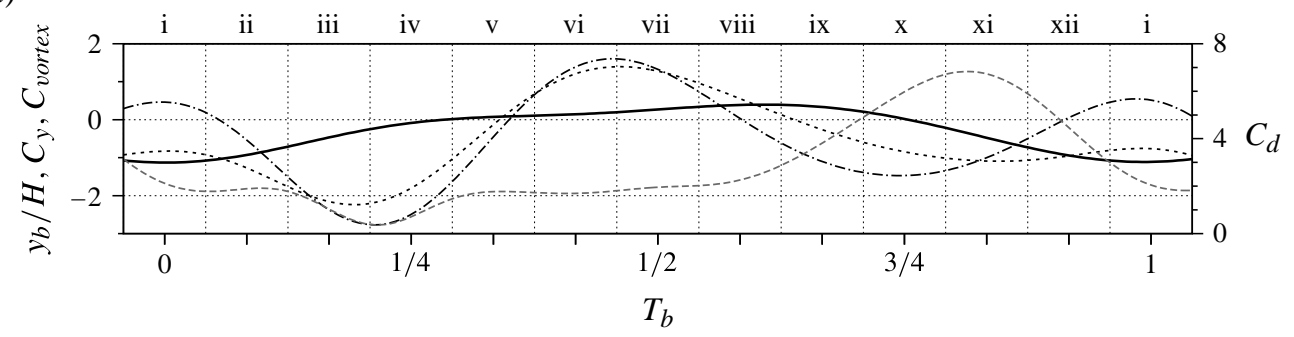

(b)
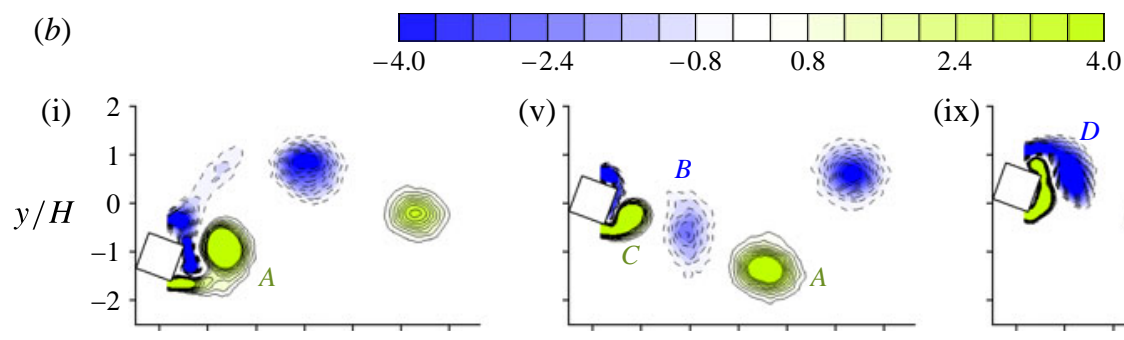

(v)
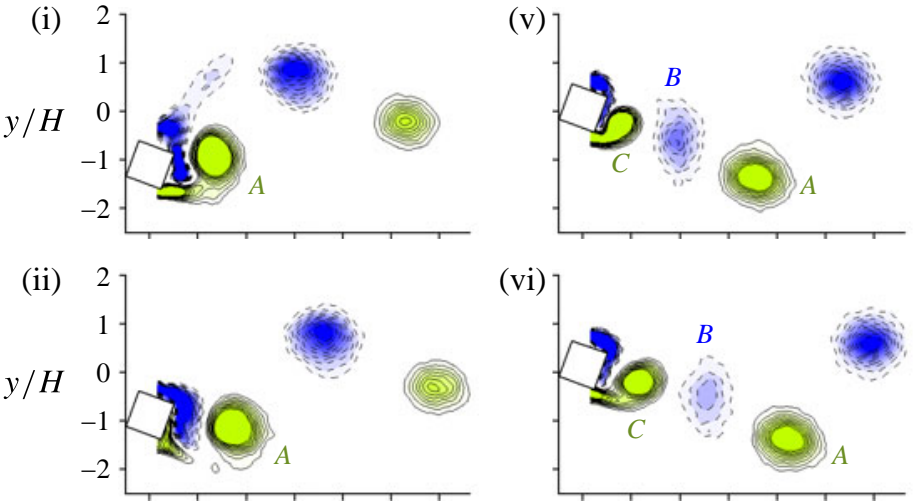

(vi)

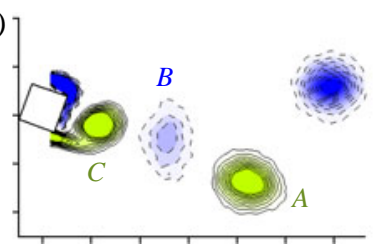

(ix)
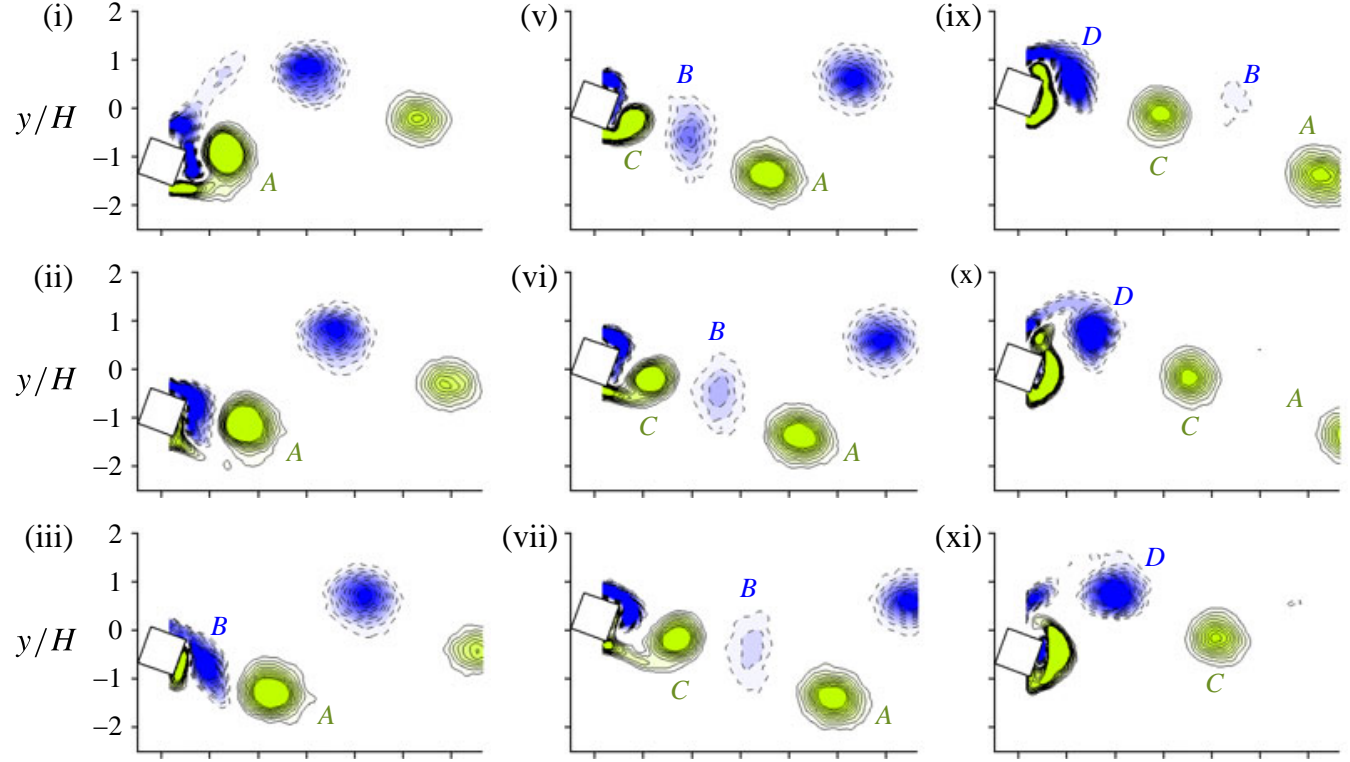

(vii)
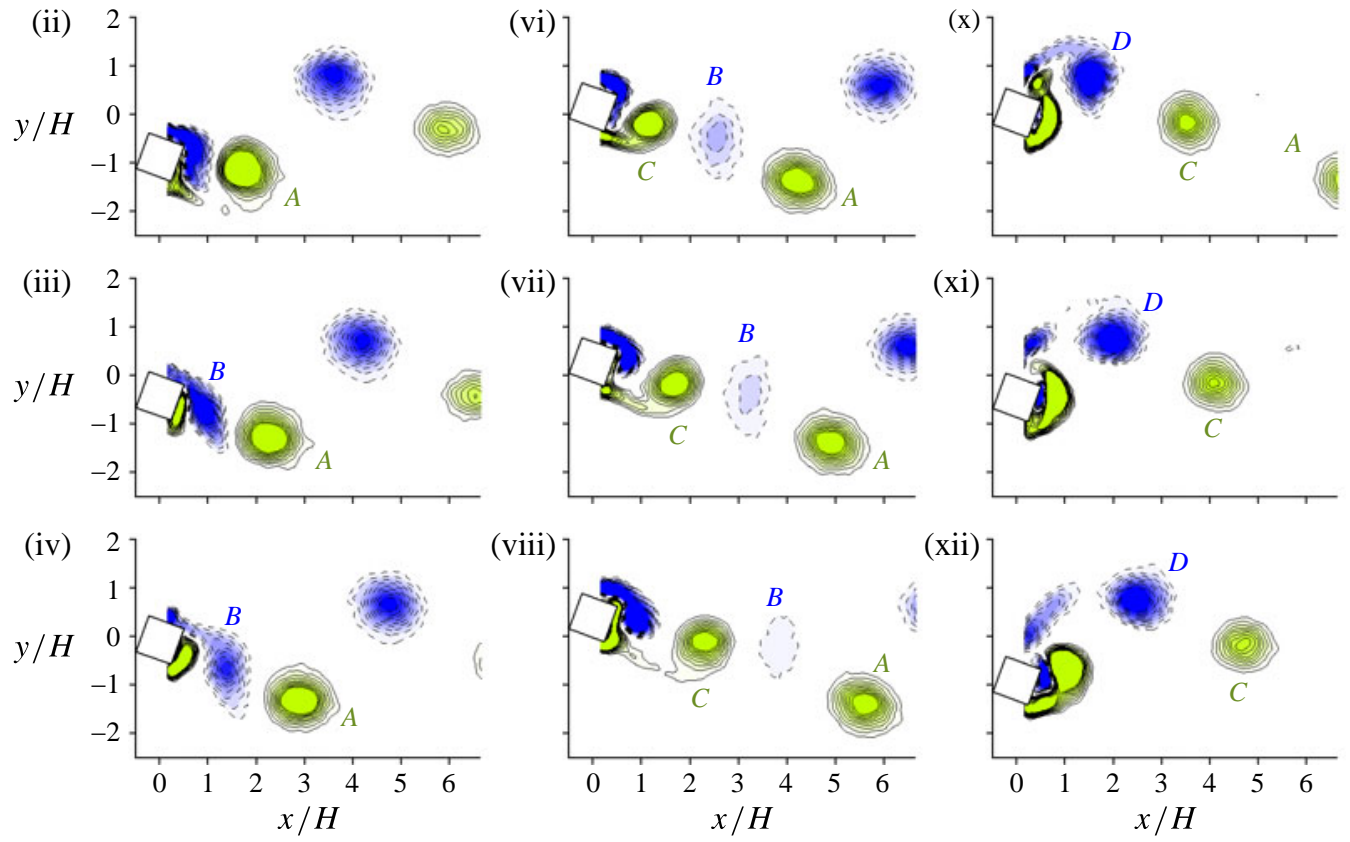

FIgURE 19. (Colour online) Time-based phase-averaged vorticity fields in the upper branch. For this case, $m^{*}=2.64$ at $\alpha=20^{\circ}$ and $U^{*}=8$. (a) The time history of the displacement and forces over a cycle of oscillation. The black solid line represents $y_{b}$, the black short-dashed line represents $C_{y}$, the black dot-dashed line represents $C_{v o r t e x}$, and the grey long-dashed line represents $C_{d}$. $(b)$ Images of vorticity from PIV averaged over the intervals marked on the time history. The vorticity contour levels shown are normalized by $\omega_{z}^{*}=\omega_{z} H / U$. The contours marked with solid lines represent positive values, and the contours marked with dashed lines represent negative values. The images show that two cycles of $2 \mathrm{~S}$ vortex shedding occur over each oscillation cycle, which has been named a $2(2 S)$ mode. 
wake over one cycle of oscillation, visualized using vorticity contours generated from PIV. Again, the images were obtained by averaging a series of PIV images over a short time. The time window used for the averaging for each image is marked on the time history shown in figure 19(a).

Figure 19 shows that two cycles of 'classic' vortex shedding (where a vortex of one sign is shed from the body in one half-cycle, then alternately a vortex of opposite sign is shed in the next half-cycle as in the Kármán vortex street) occur per oscillation cycle. Therefore, if this wake mode is to be named using the convention introduced by Williamson \& Roshko (1988), it should be designated 2(2S), meaning two cycles of two single opposite-signed vortices are shed per oscillation cycle. However, these two cycles of shedding are not identical, and there is no spatio-temporal symmetry (the second cycle is not simply the same as the first cycle reflected). The two cycles occur over different periods. This process is explained below.

As shown in figure 18, and the time history of a single cycle in figure $19(a)$, the oscillation is far from sinusoidal. Instead, the body rapidly ascends over the first quarter of a period, then spends around half an oscillation period with little variation in amplitude, before rapidly descending over the last quarter of a period.

In the first ascent phase (images $\mathrm{i}$-iii of figure 19), negative vorticity is produced at the rear surface of the body, producing a negative (dashed line) vortex attached to the trailing edge. Over this same period, the previously formed positive (solid line) vortex is shed into the wake.

At the beginning of the slow variation phase, represented by images (iv) and (v), this negative trailing-edge vortex is shed into the wake, and a new positive vortex begins to form, being fed from vorticity on the bottom surface of the body. The negative vortex shed from the trailing edge is relatively weak. Over the rest of this slow variation phase, represented by images (vi)-(viii), this positive vortex is shed into the wake, and a new negative vortex begins to form, this time fed by vorticity being produced on the top surface on the body. This new negative vortex is much stronger than the previous negative vortex that was fed by vorticity from the rear face.

During the descent phase represented by images (ix)-(xii), this negative vortex is shed into the wake, and a new positive vortex forms, this time fed by positive vorticity being generated on the rear side of the body.

In summary, the vortex shedding can be described as consisting of a pair of vortices shed during the descent and ascent, where the vortices are fed by vorticity produced at the rear surface of the body due to the acceleration of the body across the flow. This is followed by the shedding of a second pair of vortices during the phase where the body is almost stationary, the vortices this time being fed from vorticity produced at the sides of the body due to the passing flow.

Nemes et al. (2012) showed that there is an upper limit to the angle of attack $\alpha$ for the appearance of the higher branch, and consideration of the complex vorticity production mechanisms described above seems to explain this. For the vorticity production to occur at the rear surface, the surface needs to be approximately aligned with the direction of the acceleration of the body. For higher values of $\alpha$, this is not the case, and the higher branch is not observed.

The results presented in $\S 3.1 .2$ indicated that when $\alpha=0^{\circ}$, only odd numbers of vortex shedding cycles can be involved in synchronized modes. However here for $\alpha=$ $20^{\circ}$, the higher branch consists of a synchronized mode involving two shedding cycles. It is hypothesized here that this occurs due to the imbalance between the two cycles. As pointed out above, every second negative vortex is fed by vorticity from the rear face of the body and is relatively weak, whereas every other negative vortex is fed from vorticity from the top surface and is relatively strong. This difference in strength 
is enough to generate a forcing at half of the vortex shedding frequency that the body can respond to. It seems this imbalance is driven by the breaking of the reflection symmetry about the wake centreline. Here, this is done explicitly by having an angle attack. However, similar subharmonic VIV modes were observed in the simulations of a case at $\alpha=45^{\circ}$ performed by Leontini \& Thompson (2013), where the symmetry breaking was spontaneous.

\section{Conclusions}

For the case at $\alpha=0^{\circ}$, the canonical flow for the study of transverse galloping, it has been shown that the general behaviour is similar for light bodies as for the heavier bodies on which the quasi-steady theory of Parkinson \& Smith (1964) is based. However, the results of this paper for a square cylinder show that a series of 'odd' synchronizations exist, when the Strouhal frequency is in the vicinity of an odd integer multiple of the oscillation frequency. During these synchronizations, the body oscillation frequency is modified so that a multiple of the vortex shedding frequency is synchronized to the body oscillation. If the vortex shedding process is to remain similar to that of a fixed body, it has been shown that only synchronizations to odd numbers of vortex shedding cycles can occur, as synchronizations to even numbers of vortex shedding cycles do not lead to the imbalance of forces required to excite galloping.

For the case at $\alpha=45^{\circ}$, the current results show that while the motion is due to VIV, the flow response is more complicated than that of the canonical VIV case of the circular cylinder. Multiple synchronized regimes are possible, with different wake modes in each. A significant difference between the VIV of this square cylinder body and the VIV of a circular cylinder is that this body has synchronized regimes that are synchronized to the Strouhal frequency, rather than the natural frequency of the body. A small range of $U^{*}$ was also found to produce chaotic flow, which at this stage appears to be due to a very fine balance between competing forces resulting in a system that is very sensitive to external disturbances, rather than being due to any inherent chaotic dynamics.

Moving from these reflection-symmetric cases to the case at $\alpha=20^{\circ}$ leads to another set of responses. The higher branch, consisting of very high-amplitude oscillations first identified in Nemes et al. (2012), has been shown by the results presented herein to be a type of subharmonic mode, with two vortex shedding cycles per oscillation cycle. During each vortex shedding cycle, a negative vortex is shed from one side, and a positive vortex from the other, reminiscent of the classic Kármán vortex street. However, every other pair of vortices is fed from vorticity formed on the back face of the body, rather than the sides. The dependence on this back face vorticity means that this mode is only accessible for a limited range of $\alpha$. The occurrence of this subharmonic response seems to only be possible when the spatio-temporal symmetry is broken, meaning that the flow produces a non-zero mean lift. This symmetry breaking in this case is explicit, due to the angle of attack of the body.

\section{Acknowledgements}

The authors would like to acknowledge the financial support of the Australian Research Council (ARC) through grant number DP110102141 under the Discovery program. J.S.L. would like to acknowledge the financial support of the ARC through an Australian Postdoctoral Fellowship. 
Assi, G. R. S., Bearman, P. W. \& Meneghini, J. R. 2010 On the wake-induced vibration of tandem circular cylinders: the vortex interaction excitation mechanism. J. Fluid Mech. 661, $365-401$.

Bearman, P. W. 1984 Vortex shedding from oscillating bluff bodies. Annu. Rev. Fluid Mech. 16, $195-222$.

Bearman, P. W., Gartshore, I. S., Maull, D. \& Parkinson, G. V. 1987 Experiments on flow-induced vibration of a square-section cylinder. J. Fluids Struct. 1 (1), 19-34.

Blevins, R. D. 1990 Flow-Induced Vibration. 2nd edn. Krieger.

Bokaian, A. R. \& Geoola, F. 1984 Hydroelastic instabilities of square cylinders. J. Sound Vib. 92, 117-141.

Carberry, J., Sheridan, J. \& Rockwell, D. 2001 Force and wake modes of an oscillating cylinder. J. Fluids Struct. 15, 523-532.

Carberry, J., Sheridan, J. \& Rockwell, D. 2005 Controlled oscillations of a cylinder: forces and wake modes. J. Fluid Mech. 538, 31-69.

Corless, R. \& PARKinson, G. V. 1988 A model of the combined effects of vortex-induced oscillation and galloping. J. Fluids Struct. 2 (3), 203-220.

Corless, R. M. \& PARKinson, G. V. 1993 Mathematical modelling of the combined effects of vortex-induced vibration and galloping. Part II. J. Fluids Struct. 7, 825-848.

Cvitanović, P., Shraiman, B.\& Söderberg, B. 1985 Scaling laws for mode lockings in circle maps. Phys. Scr. 32 (4), 263-270.

Den Hartog, J. P. 1932 Transmission line vibration due to sleet. Trans. Am. Inst. Electr. Engrs 51 (4), 1074-1076.

Fouras, A., Lo Jacono, D. \& Hourigan, K. 2008 Target-free stereo PIV: a novel technique with inherent error estimation and improved accuracy. Exp. Fluids 44 (2), 317-329.

Govardhan, R.\& Williamson, C. H. K. 2000 Modes of vortex formation and frequency response of a freely vibrating cylinder. J. Fluid Mech. 420, 85-130.

Hahn, S. L. 1996 Hilbert Transforms in Signal Processing. Artech House.

Hover, F. S., Techet, A. H. \& Triantafyllou, M. S. 1998 Forces on oscillating uniform and tapered cylinders in crossflow. J. Fluid Mech. 363, 97-114.

Huang, N. E., Shen, Z., Long, S. R., Wu, M. C., Shih, H. H., Zheng, Q., Yen, N.-C., Tung, C. C. \& LiU, H. H. 1998 The empirical mode decomposition and the Hilbert spectrum for nonlinear and non-stationary time series analysis. Proc. R. Soc. Lond. A 454 (1971), 903-995.

Khalak, A. \& Williamson, C. H. K. 1996 Dynamics of a hydroelastic cylinder with very low mass and damping. J. Fluids Struct. 10 (5), 455-472.

Khalak, A. \& Williamson, C. H. K. 1997 Fluid forces and dynamics of a hydroelastic structure with very low mass and damping. J. Fluids Struct. 11 (8), 973-982.

Leontini, J. S., Lo Jacono, D. \& Thompson, M. C. 2011 A numerical study of an inline oscillating cylinder in a free stream. J. Fluid Mech. 688, 551-568.

Leontini, J. S., Lo JACOno, D. \& Thompson, M. C. 2013 Wake states and frequency selection of a streamwise oscillating cylinder. J. Fluid Mech. 730, 162-192.

Leontini, J. S. \& Thompson, M. C. 2013 Vortex-induced vibrations of a diamond cross-section: sensitivity to corner sharpness. J. Fluids Struct. 39, 371-390.

Lighthill, J. 1986 Fundamentals concerning wave loading on offshore structures. J. Fluid Mech. 173, 667-681.

Morse, T. L. \& Williamson, C. H. K. 2009 Prediction of vortex-induced vibration response by employing controlled motion. J. Fluid Mech. 634, 5-39.

NaUdascher, E. \& RockWell, D. 2005 Flow-Induced Vibrations: An Engineering Guide. Dover.

Nemes, A., Zhao, J., Lo JaCono, D. \& Sheridan, J. 2012 The interaction between flow-induced vibration mechanisms of a square cylinder with varying angles of attack. J. Fluid Mech. 710, $102-130$.

Norberg, C. 2001 Flow around a circular cylinder: aspects of flucuating lift. J. Fluids Struct. 15, $459-469$. 
Païdoussis, M. P., Price, S. \& De Langre, E. 2010 Fluid-Structure Interactions: Cross-FlowInduced Instabilities. Cambridge University Press.

PARKinson, G. V. \& SMITH, J. D. 1964 The square prism as an aeroelastic nonlinear oscillator. Q. J. Mech. Appl. Maths 17 (2), 225-239.

Pomeau, Y. \& ManneVille, P. 1980 Intermittent transition to turbulence in dissipative dynamical systems. Commun. Math. Phys. 74 (2), 189-197.

SARPKAYA, T. 2004 A critical review of the intrinsic nature of vortex-induced vibrations. J. Fluids Struct. 19 (4), 389-447.

Sherry, M., Lo JACONO, D. \& ShERIDAN, J. 2010 An experimental investigation of the recirculation zone formed downstream of a forward facing step. J. Wind Engng Ind. Aerodyn. 98 (12), 888-894.

Tudball-Smith, D., Leontini, J. S., Sheridan, J. \& Lo Jacono, D. 2012 Streamwise forced oscillations of circular and square cylinders. Phys. Fluids 24, 111703.

Williamson, C. H. K. \& Govardhan, R. 2004 Vortex-induced vibration. Annu. Rev. Fluid Mech. 36, 413-455.

Williamson, C. H. K. \& Roshko, A. 1988 Vortex formation in the wake of an oscillating cylinder. J. Fluids Struct. 2 (4), 355-381.

Zhao, M., Cheng, L. \& Zhou, T. 2013 Numerical simulation of vortex-induced vibration of a square cylinder at low Reynolds number. Phys. Fluids 25, 023603. 